\title{
Modular forms and three-loop superstring amplitudes
}

\author{
Sergio L. Cacciatori ${ }^{\mathrm{a}, *}$, Francesco Dalla Piazza ${ }^{\mathrm{a}}$, Bert van Geemen ${ }^{\mathrm{b}}$ \\ a Dipartimento di Scienze Fisiche e Matematiche, Università dell'Insubria, Via Valleggio 11, I-22100 Como, Italy \\ b Dipartimento di Matematica, Università di Milano, Via Saldini 50, I-20133 Milano, Italy
}

Received 9 February 2008; accepted 12 March 2008

Available online 16 March 2008

\begin{abstract}
We study a proposal of D'Hoker and Phong for the chiral superstring measure for genus three. A minor modification of the constraints they impose on certain Siegel modular forms leads to a unique solution. We reduce the problem of finding these modular forms, which depend on an even spin structure, to finding a modular form of weight 8 on a certain subgroup of the modular group. An explicit formula for this form, as a polynomial in the even theta constants, is given. We checked that our result is consistent with the vanishing of the cosmological constant. We also verified a conjecture of D'Hoker and Phong on modular forms in genus 3 and 4 using results of Igusa.

(C) 2008 Elsevier B.V. All rights reserved.
\end{abstract}

MSC: 83E30; 46T12; 14K25; 05B25

Keywords: Superstrings; Amplitudes; Modular forms; Finite geometry; Theta constants

\section{Introduction}

Formally string theories in the perturbative approach can be formulated using the path integral formalism outlined by Polyakov, and this is the starting point for the computation of the scattering amplitudes. The conformal invariance of the string theory forces the amplitude to be invariant under the action of the modular group as was exploited by Belavin and Knizhnik [1] who conjectured: "any multiloop amplitude in any conformal invariant string theory may be deduced from purely algebraic objects on moduli spaces $M_{p}$ of Riemann surfaces". Indeed, for bosonic strings

\footnotetext{
* Corresponding author.

E-mail addresses: sergio.cacciatori@uninsubria.it (S.L. Cacciatori), f.dallapiazza@uninsubria.it (F. Dalla Piazza), geemen@mat.unimi.it (B. van Geemen).
} 
this permitted the computation of the vacuum to vacuum amplitude up to four loops in terms of modular forms [2,14]. For superstrings there are some difficulties: the presence of fermionic interactions makes the splitting between chiral and antichiral modes hard, moreover one needs a covariant way to integrate out the Grassmannian variables arising from the supersymmetry on the worldsheet. In a series of articles, D'Hoker and Phong showed that the computation of $g$-loop amplitudes in string perturbation theory is strictly related to the construction of a suitable measure on the super moduli space of genus $g$ super-Riemann surfaces. They also claimed [7-9] that the genus $g$ vacuum to vacuum amplitude should take the form

$$
\mathcal{A}=\int_{\mathcal{M}_{g}}(\operatorname{det} \operatorname{Im} \tau)^{-5} \sum_{\Delta, \Delta^{\prime}} c_{\Delta, \Delta^{\prime}} \mathrm{d} \mu[\Delta](\tau) \wedge \overline{\mathrm{d} \mu\left[\Delta^{\prime}\right](\tau)}
$$

where $\Delta$ and $\Delta^{\prime}$ denote two spin structures (or theta characteristics), $c_{\Delta, \Delta^{\prime}}$ are suitable constant phases depending on the details of the model and $\mathrm{d} \mu[\Delta](\tau)$ is a holomorphic form of maximal rank $(3 g-3,0)$ on the moduli space $\mathcal{M}_{g}$ of genus $g$ Riemann surfaces. The Riemann surface is represented by its period matrix $\tau$, after a choice of canonical homology basis. Since the integrand should be independent from the choice of homology basis, it follows that the measure $\mathrm{d} \mu[\Delta](\tau)$ must transform covariantly under the modular group $\operatorname{Sp}(2 g, \mathbf{Z})$.

In [5] and following papers, D'Hoker and Phong explicitly solved the problems outlined before for the two loop vacuum to vacuum amplitude, giving an explicit expression for the two loop measure in terms of theta constants. Next, in [7,8], they tried to extend their results to three loop amplitudes. Mimicking the structure of the two loop chiral measure, they proposed three reasonable constraints (see below) which should characterize the modular forms composing the measure. Then, they tried to find such modular forms, without success. This negative result, apparently, can be imputed to their requirement that the modular form, of weight eight, should be a product of the fourth power of a theta constant and modular form of weight six. This led us to look for a weaker form of the constraints, in particular relaxing the second one, by not requiring such a decomposition and we do succeed in finding such a form. Our assumptions are consistent with the expression for the amplitudes at genus one and two, and at genus three they provide a unique solution. In this paper we will show the existence, provide an explicit expression for the measure and show that the corresponding cosmological constant is zero. For the unicity of our solution, and the fact that a solution to the constraints of D'Hoker and Phong does not exist, we refer to a future paper [4].

The constraints, and the well known one loop chiral measure, also determine the modular forms $\Xi_{6}[\delta]$ of $[5,6]$ uniquely, as we show in this paper. In particular, if one could prove a priori that the two loop chiral measure has the form indicated in Section 2.2, formula (2) with $g=2$, then we would have an easy derivation for the explicit formula of this measure.

In this paper we will use action of the modular group on modular forms as a powerful tool to solve the problem. We took advantage of the theory of induced representations: the representation furnished by the space of forms is built up from the representation given by a suitable subspace left invariant by a subgroup of the entire modular group. This approach is similar to the method that Wigner used to classify the irreducible representations of the Poincaré group induced from the representation of the little group. A systematic account of the representation of modular group on modular forms of genus three and level two will also be given in [4].

A delicate point is the assumption, made by D'Hoker and Phong and also in this paper, that 1 is true also for three loops. Moreover we will take contributions from even characteristics only. Even if there are many arguments leading to the conclusion that these last assumptions should 
not be valid for $g>2$ (see for example the discussion in [3]), we think that existence, uniqueness and simplicity of the solution are strong arguments for the opposite conclusion, at least for $g=3$. Also for $g=4$ it seems that the modified constraints have a solution, we hope to report on this in the near future. 1

The structure of the paper is the following.

In Section 2 we recall some results on the measures for the bosonic string and the chiral superstring. This leads us to consider the possibility that the chiral superstring measure might be obtained from the bosonic string measure by multiplication by a modular form of weight 8 .

In Section 2.3 we formulate some constraints which this modular form should satisfy. These constraints are very similar to the ones considered by D'Hoker and Phong in [8], the differences are discussed in Section 2.4. It turns out that for genus two we recover the chiral superstring measure as determined by D'Hoker and Phong [5,6]. In genus three our constraints have a (unique) solution.

In Section 3 we study the Siegel modular forms on $\Gamma_{g}(2)$ for $g=1,2$. In particular, we show how our constraints lead to the functions $\Xi_{6}[\delta]$ in genus two found earlier by D'Hoker and Phong. Our explicit formula for these functions in Section 3.4 is different from theirs and might be of independent interest.

In Section 4 we show that our constraints have a solution in genus three, and show that the corresponding cosmological constant is zero.

In Section 5 we briefly discuss some results of Igusa which are related to a conjecture of D'Hoker and Phong.

In Appendix A we discuss characteristics and symplectic geometry in a vector space over a field with two elements. In Appendix B we recall some facts on the transformation theory of theta constants. In Appendix C we determine the restriction of certain modular forms to 'reducible' period matrices.

\section{Measures and modular forms}

\subsection{Basic definitions}

The Siegel upper half space of complex $g \times g$ symmetric matrices with positive definite imaginary part will be denoted by $\mathbf{H}_{g}$. The action of $\Gamma_{g}:=\operatorname{Sp}(2 g, \mathbf{Z})$ on $\mathbf{H}_{g}$ is denoted as usual by

$$
M \cdot \tau:=(A \tau+B)(C \tau+D)^{-1}, \quad M:=\left(\begin{array}{cc}
A & B \\
C & D
\end{array}\right) \in \operatorname{Sp}(2 g, \mathbf{Z}), \quad \tau \in \mathbf{H}_{g} .
$$

A Siegel modular form $f$ of genus $g$ and weight $k$ on a subgroup $\Gamma \subset \operatorname{Sp}(2 g, \mathbf{Z})$ is a holomorphic function on $\mathbf{H}_{g}$ which satisfies

$$
f: \mathbf{H}_{g} \rightarrow \mathbf{C}, \quad f(M \cdot \tau)=\operatorname{det}(C \tau+D)^{k} f(\tau), \quad \forall M \in \Gamma, \tau \in \mathbf{H}_{g}
$$

(and in case $g=1$ one should also impose a growth condition on $f$ ). The factor $\operatorname{det}(C \tau+D)$ satisfies a cocycle condition:

$$
\gamma(M N, \tau)=\gamma(M, N \cdot \tau) \gamma(N, \tau), \quad \text { where } \gamma(M, \tau):=\operatorname{det}(C \tau+D) .
$$

For a subgroup $\Gamma$ of $\operatorname{Sp}(2 g, \mathbf{Z})$ which acts without fixed points on $\mathbf{H}_{g}$ one can then define a linebundle, the Hodge bundle $\lambda$, on the quotient $\Gamma \backslash \mathbf{H}_{g}$ as the quotient of the trivial bundle $\mathbf{C} \times \mathbf{H}_{g}$ on $\mathbf{H}_{g}$ by the action of $\Gamma$ given by $\gamma$ :

\footnotetext{
1 See [17,18] and also [19] for the case $g=5$.
} 


$$
\lambda:=\Gamma \backslash\left(\mathbf{C} \times \mathbf{H}_{g}\right) \rightarrow \Gamma \backslash \mathbf{H}_{g}, \quad M \cdot(t, \tau):=(\gamma(M, \tau) t, M \cdot \tau) .
$$

Even in case $\Gamma$ has fixed points, we can use this action to define a sheaf, still called $\lambda$, on $\Gamma \backslash \mathbf{H}_{g}$. The global sections of $\lambda$ correspond to the Siegel modular forms of weight 1; more generally, Siegel modular forms of weight $k$ correspond to sections of $\lambda^{\otimes k}$. For the definition of the well-known theta constants $\theta[\Delta](\tau)$ with even characteristics $\Delta$, which are modular forms of weight $1 / 2$ on a subgroup of $\operatorname{Sp}(2 g, \mathbf{Z})$, see Section 3.1. Recall that there are $2^{g-1}\left(2^{g}+1\right)$ even characteristics.

\subsection{Measures}

We recall some results on the bosonic measure for $g \leqslant 3$, the chiral superstring measure for $g \leqslant 2$ and on the proposal of D'Hoker and Phong for the chiral superstring measure for $g=3$. In this section, $c_{g}$ and $c_{g}^{\prime}$ are constants.

The genus one bosonic measure is

$$
\mathrm{d} \mu_{B}^{(1)}=\frac{1}{(2 \pi)^{12} \eta^{24}\left(\tau^{(1)}\right)} \mathrm{d} \tau^{(1)} .
$$

The genus one chiral superstring measure is (cf. e.g. [6, Eq. (8.2)])

$$
\mathrm{d} \mu\left[\Delta^{(1)}\right]=\frac{\theta\left[\Delta^{(1)}\right]^{4}\left(\tau^{(1)}\right)}{2^{5} \pi^{4} \eta^{12}\left(\tau^{(1)}\right)} \mathrm{d} \tau^{(1)},
$$

so that

$$
\mathrm{d} \mu\left[\Delta^{(1)}\right]=c_{1}^{\prime} \theta\left[\Delta^{(1)}\right]^{4}\left(\tau^{(1)}\right) \eta^{12}\left(\tau^{(1)}\right) \mathrm{d} \mu_{B}^{(1)} .
$$

Note that $\theta\left[\Delta^{(1)}\right]^{4}\left(\tau^{(1)}\right) \eta^{12}\left(\tau^{(1)}\right)$ is a modular form of weight $2+6=8$ on a subgroup of $S L(2, \mathbf{Z})$.

The genus two bosonic measure is $[2,14]$ :

$$
\mathrm{d} \mu_{B}^{(2)}=\frac{c_{2}}{\Psi_{10}\left(\tau^{(2)}\right)} \prod_{i \leqslant j} \mathrm{~d} \tau_{i j}
$$

where $\Psi_{10}$ is a modular form of weight 10 on $\operatorname{Sp}(4, \mathbf{Z})$. The genus two chiral superstring measure is (cf. $[5,6])$ :

$$
\mathrm{d} \mu\left[\Delta^{(2)}\right]=\frac{\theta\left[\Delta^{(2)}\right]^{4}\left(\tau^{(2)}\right) \Xi_{6}\left[\Delta^{(2)}\right]\left(\tau^{(2)}\right)}{16 \pi^{6} \Psi_{10}\left(\tau^{(2)}\right)} \prod_{i \leqslant j} \mathrm{~d} \tau_{i j}
$$

so that

$$
\mathrm{d} \mu\left[\Delta^{(2)}\right]=c_{2}^{\prime} \theta\left[\Delta^{(2)}\right]^{4}\left(\tau^{(2)}\right) \Xi_{6}\left[\Delta^{(2)}\right]\left(\tau^{(2)}\right) \mathrm{d} \mu_{B}^{(2)} .
$$

Note that $\theta\left[\Delta^{(2)}\right]^{4}\left(\tau^{(2)}\right) \Xi_{6}\left[\Delta^{(2)}\right]\left(\tau^{(2)}\right)$ is a modular form of weight $2+6=8$ on a subgroup of $\operatorname{Sp}(4, \mathbf{Z})$.

The genus three bosonic measure is $[2,14]$ :

$$
\mathrm{d} \mu_{B}^{(3)}=\frac{c_{3}}{\Psi_{9}\left(\tau^{(3)}\right)} \prod_{i \leqslant j} \mathrm{~d} \tau_{i j}
$$

where $\Psi_{9}^{2}\left(\tau^{(3)}\right)$ is a Siegel modular form of weight 18 for $\operatorname{Sp}(6, \mathbf{Z})$ (cf. [11] for $\left.\Psi_{9}\right)$. 
In [8, Eq. (1.1)], D'Hoker and Phong propose that the genus three chiral superstring measure is of the form

$$
\mathrm{d} \mu\left[\Delta^{(3)}\right]=\frac{\theta\left[\Delta^{(3)}\right]^{4}\left(\tau^{(3)}\right) \Xi_{6}\left[\Delta^{(3)}\right]\left(\tau^{(3)}\right)}{8 \pi^{4} \Psi_{9}\left(\tau^{(3)}\right)} \prod_{i \leqslant j} \mathrm{~d} \tau_{i j},
$$

and they give three constraints on the functions $\Xi_{6}\left[\Delta^{(3)}\right]\left(\tau^{(3)}\right)$. However, they do not succeed in finding functions which satisfy all their constraints (we will prove that there are indeed no such functions in [4]).

This leads us to weaken the proposal of [8] and to search for functions $\Xi_{8}\left[\Delta^{(3)}\right]\left(\tau^{(3)}\right)$, these should behave like the products $\theta\left[\Delta^{(3)}\right]^{4}\left(\tau^{(3)}\right) \Xi_{6}\left[\Delta^{(3)}\right]$ which occur in the numerator of $\mathrm{d} \mu\left[\Delta^{(3)}\right]$. So we assume that for $g=3$ :

$$
\mathrm{d} \mu\left[\Delta^{(g)}\right]=c_{g}^{\prime} \Xi_{8}\left[\Delta^{(g)}\right]\left(\tau^{(g)}\right) \mathrm{d} \mu_{B}^{(g)},
$$

where the functions $\Xi_{8}\left[\Delta^{(3)}\right]\left(\tau^{(3)}\right)$ satisfy three constraints which are obtained from those imposed on the functions $\theta\left[\Delta^{(3)}\right]^{4}\left(\tau^{(3)}\right) \Xi_{6}\left[\Delta^{(3)}\right]\left(\tau^{(3)}\right)$ in [8]. For example, in [8] the function $\Xi_{6}\left[\Delta^{(3)}\right]\left(\tau^{(3)}\right)$ is required to be a Siegel modular form of weight 6 on a subgroup of $\operatorname{Sp}(6, \mathbf{Z})$, and we require that $\Xi_{8}[\Delta]$ is a Siegel modular form of weight $2+6=8$ on a subgroup of $\operatorname{Sp}(6, \mathbf{Z})$. We give the three constraints on the $\Xi_{8}\left[\Delta^{(3)}\right]$ in Section 2.3. The main result of this paper is that functions $\Xi_{8}\left[\Delta^{(3)}\right]$ which satisfy all three constraints actually exist, the uniqueness of such functions will be shown in [4].

In general, one should keep in mind that since the canonical bundle on the moduli space $M_{g}$ of genus $g$ curves is $13 \lambda$, where $\lambda$ is the Hodge bundle, the bosonic measure $\mathrm{d} \mu_{B}^{(g)}$ transforms as a modular form of weight -13 (so the sections of $f \mathrm{~d} \mu_{B}^{(g)}$ of the canonical bundle correspond to modular forms $f$ of weight 13). The chiral superstring measure is known to transform as a section of $-5 \lambda$ under the action of a subgroup of $\operatorname{Sp}(2 g, \mathbf{Z})$ (see [9] and [15,16] for the supersymmetric case). Thus, taking only the transformation behaviour into account, it is not unreasonable to expect that Eq. (2) should hold for general $g$, for some function $\Xi_{8}[\Delta]$ which corresponds to a section of $8 \lambda$. As we just observed, this is proven to work for $g=1,2$ and for $g=3$ we can at least find unique $\Xi_{8}\left[\Delta^{(3)}\right]$ which satisfy reasonable constraints.

\subsection{The modular forms $\Xi_{8}[\Delta]$}

The discussion above thus leads us to search for functions

$$
\boldsymbol{\Xi}_{8}\left[\Delta^{(g)}\right]: \mathbf{H}_{g} \rightarrow \mathbf{C}, \quad \text { where } \Delta^{(g)}=\left[\begin{array}{l}
a_{1} \ldots a_{g} \\
b_{1} \ldots b_{g}
\end{array}\right]
$$

is an even characteristic, that is $a_{i}, b_{i} \in\{0,1\}$ and $\sum a_{i} b_{i} \equiv 0 \bmod 2$. It is convenient to define $\Xi_{8}\left[\Delta^{(g)}\right]=0$ in case $\Delta$ is an odd characteristic.

Actually, the $\Xi_{8}\left[\Delta^{(g)}\right]$ 's should be defined on the subvariety $J_{g} \subset \mathbf{H}_{g}$ of period matrices of Riemann surfaces of genus $g$ (note that $\operatorname{dim} J_{g}=3 g-3$ and $\operatorname{dim} \mathbf{H}_{g}=g(g+1) / 2$ ). As we do not consider the cases $g>3$ in this paper we will write $\mathbf{H}_{g}$ instead of $J_{g}$.

In order to formulate constraints for these functions for all $g$, we require that in case $g=1$ one has:

$$
\Xi_{8}\left[\Delta^{(1)}\right](\tau):=\theta\left[\Delta^{(1)}\right](\tau)^{4} \eta(\tau)^{12},
$$

see Section 2.2. Then, almost copying [8], we impose the constraints: 
(i) The functions $\Xi_{8}\left[\Delta^{(g)}\right]$ are holomorphic on $\mathbf{H}_{g}$.

(ii) Under the action of $\operatorname{Sp}(2 g, \mathbf{Z})$ on $\mathbf{H}_{g}$, these functions should transform as follows:

$$
\Xi_{8}\left[M \cdot \Delta^{(g)}\right](M \cdot \tau)=\operatorname{det}(C \tau+D)^{8} \Xi_{8}\left[\Delta^{(g)}\right](\tau),
$$

for all $M \in \operatorname{Sp}(2 g, \mathbf{Z})$, here the action of $M$ on the characteristic $\Delta$ is given by

$$
\left(\begin{array}{ll}
A & B \\
C & D
\end{array}\right) \cdot\left[\begin{array}{l}
a \\
b
\end{array}\right]:=\left[\begin{array}{l}
c \\
d
\end{array}\right], \quad\left(\begin{array}{l}
{ }^{t} c \\
{ }^{t} d
\end{array}\right)=\left(\begin{array}{cc}
D & -C \\
-B & A
\end{array}\right)\left(\begin{array}{l}
{ }^{t} a \\
{ }^{t} b
\end{array}\right)+\left(\begin{array}{c}
\left(C^{t} D\right)_{0} \\
\left(A^{t} B\right)_{0}
\end{array}\right) \bmod 2,
$$

where $N_{0}=\left(N_{11}, \ldots, N_{g g}\right)$ is the diagonal of the matrix $N$.

(iii) The restriction of these functions to 'reducible' period matrices is a product of the corresponding functions in lower genus. More precisely, let

$$
\Delta_{k, g-k}:=\left\{\tau_{k, g-k}:=\left(\begin{array}{cc}
\tau_{k} & 0 \\
0 & \tau_{g-k}
\end{array}\right) \in \mathbf{H}_{g}: \tau_{k} \in \mathbf{H}_{k}, \tau_{g-k} \in \mathbf{H}_{g-k}\right\} \cong \mathbf{H}_{k} \times \mathbf{H}_{g-k} .
$$

Then we require that for all $k, 0<k<g$,

$$
\Xi_{8}\left[\begin{array}{l}
a_{1} \ldots a_{k} a_{k+1} \ldots a_{g} \\
b_{1} \ldots b_{k} b_{k+1} \ldots b_{g}
\end{array}\right]\left(\tau_{k, g-k}\right)=\Xi_{8}\left[\begin{array}{l}
a_{1} \ldots a_{k} \\
b_{1} \ldots b_{k}
\end{array}\right]\left(\tau_{k}\right) \Xi_{8}\left[\begin{array}{l}
a_{k+1} \ldots a_{g} \\
b_{k+1} \ldots b_{g}
\end{array}\right]\left(\tau_{g-k}\right)
$$

for all even characteristics $\Delta^{(g)}=\left[\begin{array}{l}a_{1} \ldots a_{g} \\ b_{1} \ldots b_{g}\end{array}\right]$ and all $\tau_{k, g-k} \in \Delta_{k, g-k}$.

\subsection{Remark: Comparison with [8]}

We compare these constraints with those of D'Hoker and Phong in [8] for the functions $\Xi_{6}[\Delta]$ on $\mathbf{H}_{3}$. The only essential difference is in constraint (ii). Note that the products $\theta[\Delta]^{4}(\tau) \Xi_{6}[\Delta](\tau)$, with $\Xi_{6}[\Delta]$ as in their constraint (ii) and $\tau \in \mathbf{H}_{3}$, transforms in the same way as our $\Xi_{8}[\Delta]$ but with a factor $\epsilon(M, \Delta)^{4+4}$. However $\epsilon(M, \Delta)^{8}=1$, so $\theta[\Delta]^{4}(\tau) \Xi_{6}[\Delta](\tau)$ transforms as $\Xi_{8}[\Delta]$. Conversely, if each $\Xi_{8}[\Delta]$ were a product of $\theta[\Delta]^{4}$ and another function, these other functions would satisfy constraint (ii) of [8].

\subsection{Remark on condition (ii)}

Let $\Gamma_{g}(2)$ be the (normal) subgroup of $\operatorname{Sp}(2 g, \mathbf{Z})$ defined by:

$$
\begin{aligned}
\Gamma_{g}(2) & =\operatorname{ker}\left(\operatorname{Sp}(2 g, \mathbf{Z}) \rightarrow \operatorname{Sp}\left(2 g, \mathbf{F}_{2}\right)\right) \\
& =\{M \in \operatorname{Sp}(2 g, \mathbf{Z}): A \equiv D \equiv I, B \equiv C \equiv 0 \bmod 2\},
\end{aligned}
$$

where we write $\mathbf{F}_{2}:=\mathbf{Z} / 2 \mathbf{Z}$ for the field with two elements. For $M \in \Gamma_{g}(2)$ we have $M \cdot\left[\begin{array}{l}a \\ b\end{array}\right]=$ $\left[\begin{array}{l}a \\ b\end{array}\right]$ for all characteristics $\left[\begin{array}{l}a \\ b\end{array}\right]$, hence the $\Xi_{8}\left[\Delta^{(g)}\right]$ are modular forms of genus $g$ and weight 8 on $\Gamma_{g}(2)$.

\subsection{Remark on condition (iii)}

In [8], the third constraint is imposed for an arbitrary separating degeneration. However, any such degeneration is obtained from the one in condition (iii) by a symplectic transformation. Thus one has to consider the functions $\Xi_{8}[\Delta]\left(N \cdot \tau_{k, g-k}\right)$ for all $N \in \operatorname{Sp}(2 g, \mathbf{Z})$. Constraint (ii) shows that this amounts to considering $\Xi_{8}\left[N^{-1} \cdot \Delta\right]\left(\tau_{k, g-k}\right)$ (up to an easy factor) and constraint (iii) determines this function. 


\subsection{Reduction to the case $\left[\Delta^{(g)}\right]=\left[\begin{array}{l}0 \\ 0\end{array}\right]$}

The second constraint, in particular Eq. (3), can be used to restrict the search for the $2^{g-1}\left(2^{g}+1\right)$ functions $\Xi_{8}\left[\Delta^{(g)}\right]$ to that of a single one, for which we choose $\Xi_{8}\left[\begin{array}{l}0 \\ 0\end{array}\right]$ with $\left[\begin{array}{l}0 \\ 0\end{array}\right]=\left[\begin{array}{c}0 \ldots 0 \\ 0 \ldots 0\end{array}\right]$. We work out the details of this reduction. In particular, we give the constraints which the function $\Xi_{8}\left[\begin{array}{l}0 \\ 0\end{array}\right]$ should satisfy and given this function we define functions $\Xi_{8}\left[\Delta^{(g)}\right]$, for all even characteristics $\Delta^{(g)}$, which satisfy the constraints from Section 2.3.

Let $\Gamma_{g}(1,2)$ be the subgroup of $\operatorname{Sp}(2 g, \mathbf{Z})$ which fixes the characteristic $\left[\begin{array}{l}0 \\ 0\end{array}\right]:=\left[\begin{array}{c}0 \ldots 0 \\ 0 \ldots 0\end{array}\right]$ :

$$
\begin{aligned}
\Gamma_{g}(1,2) & :=\left\{M \in \Gamma_{g}: M \cdot\left[\begin{array}{c}
0 \\
0
\end{array}\right] \equiv\left[\begin{array}{l}
0 \\
0
\end{array}\right] \bmod 2\right\} \\
& =\left\{M \in \Gamma_{g}: \operatorname{diag} A^{t} B \equiv \operatorname{diag} C^{t} D \equiv 0 \bmod 2\right\} .
\end{aligned}
$$

For $M \in \Gamma_{g}(1,2)$ we required that $\Xi_{8}\left[\begin{array}{l}0 \\ 0\end{array}\right](M \cdot \tau)=(C \tau+D)^{8} \Xi_{8}\left[\begin{array}{l}0 \\ 0\end{array}\right](\tau)$, that is, $\Xi_{8}\left[\begin{array}{l}0 \\ 0\end{array}\right]$ is a modular form on $\Gamma_{g}(1,2)$ of weight 8 .

Given such a modular form $\Xi_{8}\left[\begin{array}{l}0 \\ 0\end{array}\right]$ on $\Gamma_{g}(1,2)$ we now define, for each even characteristic $\Delta$ a function $\Xi_{8}[\Delta]$ in such a way that Eq. (3) holds. As the group $\operatorname{Sp}(2 g, \mathbf{Z})$ acts transitively on the even characteristics, for any even characteristic $\left[\Delta^{(g)}\right]$ there is an $M \in \operatorname{Sp}(2 g, \mathbf{Z})$ with $M \cdot\left[\begin{array}{l}0 \\ 0\end{array}\right]=\left[\Delta^{(g)}\right] \bmod 2$ and then we define, with $\gamma$ as in Section 2.1,

$$
\Xi_{8}\left[\Delta^{(g)}\right](\tau):=\gamma\left(M, M^{-1} \cdot \tau\right)^{8} \Xi_{8}\left[\begin{array}{c}
0 \\
0
\end{array}\right]\left(M^{-1} \cdot \tau\right) .
$$

It is easy to check that the definition of $\Xi_{8}\left[\Delta^{(g)}\right]$ does not depend on the choice of $M$ : if also $N \cdot\left[\begin{array}{l}0 \\ 0\end{array}\right]=\left[\Delta^{(g)}\right] \bmod 2$, then $N^{-1} M$ fixes $\left[\begin{array}{l}0 \\ 0\end{array}\right]$ so $N^{-1} M \in \Gamma_{g}(1,2)$. To verify that

$$
\gamma\left(M, M^{-1} \cdot \tau\right)^{8} \Xi_{8}\left[\begin{array}{l}
0 \\
0
\end{array}\right]\left(M^{-1} \cdot \tau\right) \stackrel{?}{=} \gamma\left(N, N^{-1} \cdot \tau\right)^{8} \Xi_{8}\left[\begin{array}{l}
0 \\
0
\end{array}\right]\left(N^{-1} \cdot \tau\right)
$$

we let $\tau=M \tau^{\prime}$, so we must verify that

$$
\gamma\left(M, \tau^{\prime}\right)^{8} \Xi_{8}\left[\begin{array}{l}
0 \\
0
\end{array}\right]\left(\tau^{\prime}\right) \stackrel{?}{=} \gamma\left(N, N^{-1} M \cdot \tau^{\prime}\right)^{8} \Xi_{8}\left[\begin{array}{l}
0 \\
0
\end{array}\right]\left(N^{-1} M \cdot \tau^{\prime}\right) .
$$

As $N^{-1} M \in \Gamma_{g}(1,2)$ and $\gamma$ satisfies the cocycle condition, we get

$$
\begin{aligned}
\gamma\left(N, N^{-1} M \cdot \tau^{\prime}\right)^{8} \Xi_{8}\left[\begin{array}{l}
0 \\
0
\end{array}\right]\left(N^{-1} M \cdot \tau^{\prime}\right) & =\gamma\left(N, N^{-1} M \cdot \tau^{\prime}\right)^{8} \gamma\left(N^{-1} M, \tau^{\prime}\right)^{8} \Xi_{8}\left[\begin{array}{l}
0 \\
0
\end{array}\right]\left(\tau^{\prime}\right) \\
& =\gamma\left(M, \tau^{\prime}\right)^{8} \Xi_{8}\left[\begin{array}{l}
0 \\
0
\end{array}\right]\left(\tau^{\prime}\right),
\end{aligned}
$$

which verifies the desired identity. Finally we show that the functions $\Xi_{8}\left[\Delta^{(g)}\right]$ satisfy constraint (ii) of Section 2.3. So with $M, \Delta^{(g)}$ as above, we must verify that for all $N \in \operatorname{Sp}(2 g, \mathbf{Z})$ we have

$$
\Xi_{8}[N \cdot \Delta](N \cdot \tau) \stackrel{?}{=} \gamma(N, \tau)^{8} \Xi_{8}[\Delta](\tau) .
$$

As $N \cdot \Delta=N M \cdot\left[\begin{array}{l}0 \\ 0\end{array}\right]$, we have:

$$
\begin{aligned}
\Xi_{8}[N \cdot \Delta](N \cdot \tau) & =\gamma\left(N M,(N M)^{-1} N \cdot \tau\right)^{8} \Xi_{8}\left[\begin{array}{l}
0 \\
0
\end{array}\right]\left((N M)^{-1} N \cdot \tau\right) \\
& =\gamma\left(N M, M^{-1} \cdot \tau\right)^{8} \Xi_{8}\left[\begin{array}{l}
0 \\
0
\end{array}\right]\left(M^{-1} \tau\right) \\
& =\gamma(N, \tau)^{8} \gamma\left(M, M^{-1} \cdot \tau\right)^{8} \Xi_{8}\left[\begin{array}{l}
0 \\
0
\end{array}\right]\left(M^{-1} \cdot \tau\right) \\
& =\gamma(N, \tau)^{8} \Xi_{8}[\Delta](\tau),
\end{aligned}
$$


where we used the cocycle relation. Thus the second constraint is verified if $\Xi_{8}\left[\begin{array}{l}0 \\ 0\end{array}\right]$ satisfies the constraint (ii 0 ) below and if the $\Xi_{8}\left[\Delta^{(g)}\right]$ are defined as in Eq. (4).

(ii 0 ) The function $\Xi_{8}\left[\begin{array}{l}0 \\ 0\end{array}\right]$ is a modular form $\Xi_{8}$ of weight 8 on $\Gamma_{g}(1,2)$.

Next we show that, in case $g \leqslant 3$, constraint (iii) follows from the constraints:

(iii 0 ) (1) For all $k, 0<k<g$, and all $\tau_{k, g-k} \in \Delta_{k, g-k}$ we have

$$
\Xi_{8}\left[\begin{array}{l}
0 \\
0
\end{array}\right]\left(\tau_{k, g-k}\right)=\Xi_{8}\left[\begin{array}{l}
0 \\
0
\end{array}\right]\left(\tau_{k}\right) \Xi_{8}\left[\begin{array}{l}
0 \\
0
\end{array}\right]\left(\tau_{g-k}\right) .
$$

(iii 0 ) (2) If $\Delta^{(g)}=\left[\begin{array}{l}a b \ldots \\ c d \ldots]\end{array}\right]$ with $a c=1$ then $\Xi_{8}\left[\Delta^{(g)}\right]\left(\tau_{1, g-1}\right)=0$.

Obviously (iii) implies (iii $)$ (1), (2). We will only show how to use (iii $\left.{ }_{0}\right)(1)$. Let

$$
\Delta^{(g)}=\left[\begin{array}{l}
a_{1} \ldots a_{g} \\
b_{1} \ldots b_{g}
\end{array}\right], \quad \Delta^{(k)}:=\left[\begin{array}{l}
a_{1} \ldots a_{k} \\
b_{1} \ldots b_{k}
\end{array}\right], \quad \Delta^{(g-k)}:=\left[\begin{array}{l}
a_{k+1} \ldots a_{g} \\
b_{k+1} \ldots b_{g}
\end{array}\right]
$$

and assume that $\Delta^{(k)}$ is even, then also $\Delta^{(g-k)}$ is even. Thus there are symplectic matrices $M_{1} \in$ $\operatorname{Sp}(2 k, \mathbf{Z})$ and $M_{2} \in S p(2(g-k), \mathbf{Z})$ such that $M_{1} \cdot\left[\begin{array}{l}0 \\ 0\end{array}\right]=\left[\Delta^{(k)}\right]$ and $M_{2} \cdot\left[\begin{array}{l}0 \\ 0\end{array}\right]=\left[\Delta^{(g-k)}\right]$. Hence the matrix $M \in \operatorname{Sp}(2 g, \mathbf{Z})$ obtained from $M_{1}, M_{2}$ in the obvious way has the properties: $M$. $\left(\Delta_{k, g-k}\right)=\Delta_{k, g-k}$ and $\Delta^{(g)}=M \cdot\left[\begin{array}{l}0 \\ 0\end{array}\right]$. As $M, \tau_{k, g-k}$ are made up of $k \times k$ and $(g-k) \times(g-k)$ blocks one has

$$
\gamma\left(M, M^{-1} \cdot \tau_{k, g-k}\right)=\gamma\left(M_{1}, M_{1}^{-1} \cdot \tau_{k}\right) \gamma\left(M_{2}, M_{2}^{-1} \cdot \tau_{g-k}\right), \quad \Delta^{(g)}=M \cdot\left[\begin{array}{l}
0 \\
0
\end{array}\right] .
$$

Moreover, $M^{-1} \cdot \tau_{k, g-k}$ is the matrix in $\Delta_{k, g-k}$ with blocks $M_{1}^{-1} \cdot \tau_{k}$ and $M_{2}^{-1} \cdot \tau_{g-k}$. Thus if (iii) $)$ (1) is satisfied we have:

$$
\Xi_{8}\left[\begin{array}{l}
0 \\
0
\end{array}\right]\left(M^{-1} \cdot \tau_{k, g-k}\right)=\Xi_{8}\left[\begin{array}{c}
0 \\
0
\end{array}\right]\left(M_{1}^{-1} \cdot \tau_{k}\right) \Xi_{8}\left[\begin{array}{l}
0 \\
0
\end{array}\right]\left(M_{2}^{-1} \cdot \tau_{g-k}\right) .
$$

Then we have:

$$
\begin{aligned}
\Xi_{8}\left[\Delta^{(g)}\right]\left(\tau_{k, g-k}\right)= & \gamma\left(M, M^{-1} \cdot \tau\right)^{8} \Xi_{8}\left[\begin{array}{l}
0 \\
0
\end{array}\right]\left(M^{-1} \cdot \tau_{k, g-k}\right) \\
= & \gamma\left(M_{1}, M_{1}^{-1} \cdot \tau_{k}\right)^{8} \gamma\left(M_{2}, M_{2}^{-1} \cdot \tau_{g-k}\right)^{8} \Xi_{8}\left[\begin{array}{l}
0 \\
0
\end{array}\right]\left(M_{1}^{-1} \cdot \tau_{k}\right) \\
& \times \Xi_{8}\left[\begin{array}{l}
0 \\
0
\end{array}\right]\left(M_{2}^{-1} \cdot \tau_{g-k}\right) \\
= & \Xi_{8}\left[\Delta^{(k)}\right]\left(\tau_{k}\right) \Xi_{8}\left[\Delta^{(g-k)}\right]\left(\tau_{g-k}\right),
\end{aligned}
$$

so for these $\Delta^{(g)}$ the functions $\Xi_{8}\left[\Delta^{(g)}\right]$ satisfy (iii).

\section{Siegel modular forms}

\subsection{Theta constants}

Modular forms of even weight on $\Gamma_{g}(2)$ can be obtained as products of the even theta constants

$$
\theta\left[\begin{array}{l}
a \\
b
\end{array}\right](\tau):=\sum_{m \in \mathbf{Z}^{g}} e^{\pi i\left(^{t}(m+a / 2) \tau(m+a / 2)+{ }^{t}(m+a / 2) b\right)}
$$

with $a=\left(a_{1}, \ldots, a_{g}\right), b=\left(b_{1}, \ldots, b_{g}\right), a_{i}, b_{i} \in\{0,1\}$ and $\sum a_{i} b_{i} \equiv 0 \bmod 2$ (note that we write $a, b$ as row vectors in $\left[\begin{array}{l}a \\ b\end{array}\right]$ but as column vectors in $\left.m+a / 2,{ }^{t}(m+a / 2) b\right)$. These theta constants are modular forms, of weight $1 / 2$, for a subgroup of $\operatorname{Sp}(2 g, \mathbf{Z})$. 
It is convenient to define the $2^{g}$ theta constants:

$$
\Theta[\sigma](\tau):=\theta\left[\begin{array}{l}
\sigma \\
0
\end{array}\right](2 \tau), \quad[\sigma]=\left[\begin{array}{llll}
\sigma_{1} & \sigma_{2} & \cdots & \sigma_{g}
\end{array}\right], \sigma_{i} \in\{0,1\}, \tau \in \mathbf{H}_{g} .
$$

The $\Theta[\sigma]$ have the advantage that they are algebraically independent for $g \leqslant 2$ and there is a unique relation of degree 16 for $g=3$, whereas there are many algebraic relations between the $\theta[\Delta]$ 's, for example Jacobi's relation in $g=1$.

\subsection{A classical formula}

A classical formula for theta functions shows that any $\theta[\Delta]^{2}$ is a linear combination of products of two $\Theta[\sigma]$ 's. Note that there are $2^{g}$ functions $\Theta[\sigma]$ and thus there are $\left(2^{g}+1\right) 2^{g} / 2=$ $2^{g-1}\left(2^{g}+1\right)$ products $\Theta[\sigma] \Theta\left[\sigma^{\prime}\right]$. This is also the number of even characteristics, and the products $\Theta[\sigma] \Theta\left[\sigma^{\prime}\right]$ span the same space (of modular forms of weight 1 ) as the $\theta[\Delta]^{2}$,s.

The classical formula used here is (cf. [12, IV.1, Theorem 2]):

$$
\theta\left[\begin{array}{c}
\epsilon \\
\epsilon^{\prime}
\end{array}\right]^{2}=\sum_{\sigma}(-1)^{\sigma \epsilon^{\prime}} \Theta[\sigma] \Theta[\sigma+\epsilon]
$$

where we sum over the $2^{g}$ vectors $\sigma$ and $\left[\begin{array}{c}\epsilon \\ \epsilon^{\prime}\end{array}\right]$ is an even characteristic, so $\epsilon \epsilon^{\prime} \equiv 0 \bmod 2$. These formulae are easily inverted to give:

$$
\Theta[\sigma] \Theta[\sigma+\epsilon]=\frac{1}{2^{g}} \sum_{\epsilon^{\prime}}(-1)^{\sigma \epsilon^{\prime}} \theta\left[\begin{array}{c}
\epsilon \\
\epsilon^{\prime}
\end{array}\right]^{2} .
$$

\subsubsection{Example}

In case $g=1$ one has

$$
\theta\left[\begin{array}{l}
0 \\
0
\end{array}\right]^{2}=\Theta[0]^{2}+\Theta[1]^{2}, \quad \theta\left[\begin{array}{l}
0 \\
1
\end{array}\right]^{2}=\Theta[0]^{2}-\Theta[1]^{2}, \quad \theta\left[\begin{array}{l}
1 \\
0
\end{array}\right]^{2}=2 \Theta[0] \Theta[1],
$$

or, equivalently,

$$
\begin{aligned}
& \Theta[0]^{2}=\left(\theta\left[\begin{array}{l}
0 \\
0
\end{array}\right]^{2}+\theta\left[\begin{array}{l}
0 \\
1
\end{array}\right]^{2}\right) / 2, \quad \Theta[1]^{2}=\left(\theta\left[\begin{array}{l}
0 \\
0
\end{array}\right]^{2}-\theta\left[\begin{array}{l}
0 \\
1
\end{array}\right]^{2}\right) / 2, \\
& \Theta[0] \Theta[1]=\theta\left[\begin{array}{l}
1 \\
0
\end{array}\right]^{2} / 2 .
\end{aligned}
$$

Note that upon substituting the first three relations in Jacobi's relation $\theta\left[\begin{array}{l}0 \\ 0\end{array}\right]^{4}=\theta\left[\begin{array}{l}0 \\ 1\end{array}\right]^{4}+\theta\left[\begin{array}{l}1 \\ 0\end{array}\right]^{4}$ one obtains a trivial identity in the $\Theta[\sigma]$ 's.

\subsection{The case $g=1$}

In the genus one case, the modular forms $\Xi_{8}[\Delta]$ are given by $\Xi_{8}[\Delta]=\theta[\Delta]^{4} \eta^{12}$. A classical formula for the Dedekind $\eta$ function is: $\eta^{3}=\theta\left[\begin{array}{l}0 \\ 0\end{array}\right] \theta\left[\begin{array}{l}0 \\ 1\end{array}\right] \theta\left[\begin{array}{l}1 \\ 0\end{array}\right]$, so

$$
\begin{aligned}
\eta^{12} & =\theta\left[\begin{array}{l}
0 \\
0
\end{array}\right]^{4} \theta\left[\begin{array}{l}
0 \\
1
\end{array}\right]^{4} \theta\left[\begin{array}{l}
1 \\
0
\end{array}\right]^{4} \\
& =\left(\Theta[0]^{2}+\Theta[1]^{2}\right)^{2}\left(\Theta[0]^{2}-\Theta[1]^{2}\right)^{2}(2 \Theta[0] \Theta[1])^{2} .
\end{aligned}
$$

Another useful formula for $\eta^{12}$ is closely related to Jacobi's relation:

$$
3 \eta^{12}=\theta\left[\begin{array}{l}
0 \\
0
\end{array}\right]^{12}-\theta\left[\begin{array}{l}
0 \\
1
\end{array}\right]^{12}-\theta\left[\begin{array}{l}
1 \\
0
\end{array}\right]^{12},
$$


it suffices to use the classical formula's, expressing the $\theta\left[\begin{array}{l}a \\ b\end{array}\right]$ 's in the $\Theta[\sigma]$ 's, to verify the identity.

The function $\Xi_{8}\left[\begin{array}{l}0 \\ 0\end{array}\right]=\theta\left[\begin{array}{l}0 \\ 0\end{array}\right]^{4} \eta^{12}$ is a modular form on $\Gamma_{1}(1,2)$ of weight eight. Another modular form of the same type is $\theta\left[\begin{array}{l}0 \\ 0\end{array}\right]^{4} f_{21}(\tau)$ with

$$
f_{21}:=2 \theta\left[\begin{array}{l}
0 \\
0
\end{array}\right]^{12}+\theta\left[\begin{array}{l}
0 \\
1
\end{array}\right]^{12}+\theta\left[\begin{array}{l}
1 \\
0
\end{array}\right]^{12} \text {. }
$$

Below we write some other modular forms, which we will need later, in terms of $f_{21}, \eta^{12}$ :

$$
\begin{aligned}
& \theta^{12}\left[\begin{array}{l}
0 \\
0
\end{array}\right]=\frac{1}{3} f_{21}+\eta^{12} \\
& \theta\left[\begin{array}{l}
0 \\
0
\end{array}\right]^{4}\left(\theta\left[\begin{array}{l}
0 \\
0
\end{array}\right]^{8}+\theta\left[\begin{array}{l}
0 \\
1
\end{array}\right]^{8}+\theta\left[\begin{array}{l}
1 \\
0
\end{array}\right]^{8}\right)=\theta\left[\begin{array}{l}
0 \\
0
\end{array}\right]^{12}+\theta\left[\begin{array}{l}
0 \\
0
\end{array}\right]^{4}\left(\theta\left[\begin{array}{l}
0 \\
0
\end{array}\right]^{8}-2 \theta\left[\begin{array}{l}
0 \\
1
\end{array}\right]^{4} \theta\left[\begin{array}{l}
1 \\
0
\end{array}\right]^{4}\right) \\
& =\left(\frac{1}{3} f_{21}+\eta^{12}\right)+\left(\frac{1}{3} f_{21}+\eta^{12}\right)-2 \eta^{12} \\
& =\frac{2}{3} f_{21} \text {, } \\
& \theta\left[\begin{array}{l}
0 \\
0
\end{array}\right]^{12}+\theta\left[\begin{array}{l}
0 \\
1
\end{array}\right]^{12}+\theta\left[\begin{array}{l}
1 \\
0
\end{array}\right]^{12}=f_{21}-\theta\left[\begin{array}{l}
0 \\
0
\end{array}\right]^{12} \\
& =\frac{2}{3} f_{21}-\eta^{12} \text {, } \\
& \theta\left[\begin{array}{l}
0 \\
0
\end{array}\right]^{4} \theta\left[\begin{array}{l}
0 \\
1
\end{array}\right]^{8}+\theta\left[\begin{array}{l}
0 \\
0
\end{array}\right]^{4} \theta\left[\begin{array}{l}
1 \\
0
\end{array}\right]^{8}=\theta\left[\begin{array}{l}
0 \\
0
\end{array}\right]^{4}\left(\theta\left[\begin{array}{l}
0 \\
0
\end{array}\right]^{8}+\theta\left[\begin{array}{l}
0 \\
1
\end{array}\right]^{8}+\theta\left[\begin{array}{l}
1 \\
0
\end{array}\right]^{8}\right)-\theta\left[\begin{array}{l}
0 \\
0
\end{array}\right]^{12} \\
& =\frac{2}{3} f_{21}-\left(\frac{1}{3} f_{21}+\eta^{12}\right) \\
& =\frac{1}{3} f_{21}-\eta^{12} \text {. }
\end{aligned}
$$

\subsection{The case $g=2$}

In case $g=2$, we define three holomorphic functions on $\mathbf{H}_{2}$ :

$$
f_{1}:=\theta\left[\begin{array}{c}
00 \\
00
\end{array}\right]^{12}, \quad f_{2}:=\sum_{\delta} \theta[\delta]^{12}, \quad f_{3}:=\theta\left[\begin{array}{c}
00 \\
00
\end{array}\right]^{4} \sum_{\delta} \theta[\delta]^{8},
$$

where we sum over the 10 even characteristics $\delta$ in genus 2 . The functions $\theta\left[\begin{array}{c}00 \\ 00\end{array}\right]^{4} f_{i}, i=1,2,3$, are modular forms of weight 8 for $\Gamma_{2}(1,2)$, see Appendix B.3.

The function $\sum_{\delta} \theta[\delta]^{16}$ is a modular form on $\operatorname{Sp}(4, \mathbf{Z})$, and hence is a modular form of weight 8 for $\Gamma_{2}(1,2)$, but we do not need it. In [4] we will show that the three $\Theta\left[\begin{array}{c}00 \\ 00\end{array}\right]^{4} f_{i}$ and $\sum_{\delta} \theta[\delta]^{16}$ are a basis of the modular forms of weight 8 on $\Gamma_{2}(1,2)$.

The third constraint on the function $\Xi_{8}\left[\begin{array}{c}00 \\ 00\end{array}\right]$ is:

$$
\Xi_{8}\left[\begin{array}{c}
00 \\
00
\end{array}\right]\left(\tau_{1,1}\right)=\left(\theta\left[\begin{array}{l}
0 \\
0
\end{array}\right]^{4} \eta^{12}\right)\left(\tau_{1}\right)\left(\theta\left[\begin{array}{l}
0 \\
0
\end{array}\right]^{4} \eta^{12}\right)\left(\tau_{1}^{\prime}\right)
$$

where $\tau_{1,1}=\operatorname{diag}\left(\tau_{1}, \tau_{1}^{\prime}\right)$ and $\tau_{1}, \tau_{1}^{\prime} \in \mathbf{H}_{1}$. We try to determine $a_{i} \in \mathbf{C}$ such that $\theta\left[\begin{array}{c}00 \\ 00\end{array}\right]^{4} \sum_{i} a_{i} f_{i}$ factors in this way for such period matrices. Note that

$$
\theta\left[\begin{array}{l}
a b \\
c d
\end{array}\right]\left(\tau_{1,1}\right)=\theta\left[\begin{array}{l}
a \\
c
\end{array}\right]\left(\tau_{1}\right) \theta\left[\begin{array}{l}
b \\
d
\end{array}\right]\left(\tau_{1}^{\prime}\right)
$$


in particular $\theta\left[\begin{array}{l}a b \\ c d\end{array}\right]\left(\tau_{1,1}\right)=0$ if $a c=1$. As $\theta\left[\begin{array}{c}00 \\ 00\end{array}\right]\left(\tau_{1,1}\right)$ produces $\theta\left[\begin{array}{l}0 \\ 0\end{array}\right]^{4}\left(\tau_{1}\right) \theta\left[\begin{array}{l}0 \\ 0\end{array}\right]^{4}\left(\tau_{1}^{\prime}\right)$, it remains to find $a_{i}$ such that

$$
\eta^{12}\left(\tau_{1}\right) \eta^{12}\left(\tau_{1}^{\prime}\right)=\left(a_{1} f_{1}+a_{2} f_{2}+a_{3} f_{3}\right)\left(\tau_{1,1}\right) .
$$

Using the results from 3.3, the restrictions of the $f_{i}$ are:

$$
\begin{gathered}
\theta\left[\begin{array}{l}
00 \\
00
\end{array}\right]^{12}\left(\tau_{1,1}\right)=\theta\left[\begin{array}{l}
0 \\
0
\end{array}\right]^{12}\left(\tau_{1}\right) \theta\left[\begin{array}{l}
0 \\
0
\end{array}\right]^{12}\left(\tau_{1}^{\prime}\right) \\
=\left(\frac{1}{3} f_{21}+\eta^{12}\right)\left(\tau_{1}\right)\left(\frac{1}{3} f_{21}+\eta^{12}\right)\left(\tau_{1}^{\prime}\right) \\
\left(\sum_{\delta} \theta[\delta]^{12}\right)\left(\tau_{1,1}\right)=\left(\theta\left[\begin{array}{l}
0 \\
0
\end{array}\right]^{12}+\theta\left[\begin{array}{l}
0 \\
1
\end{array}\right]^{12}+\theta\left[\begin{array}{l}
1 \\
0
\end{array}\right]^{12}\right)\left(\tau_{1}\right)\left(\theta\left[\begin{array}{l}
0 \\
0
\end{array}\right]^{12}+\theta\left[\begin{array}{l}
0 \\
1
\end{array}\right]^{12}+\theta\left[\begin{array}{l}
1 \\
0
\end{array}\right]^{12}\right)\left(\tau_{1}^{\prime}\right) \\
=\left(\frac{2}{3} f_{21}-\eta^{12}\right)\left(\tau_{1}\right)\left(\frac{2}{3} f_{21}-\eta^{12}\right)\left(\tau_{1}^{\prime}\right) \\
\left(\theta\left[\begin{array}{c}
00 \\
00
\end{array}\right]^{4} \sum_{\delta} \theta[\delta]^{8}\right)\left(\tau_{1,1}\right)=\left(\theta\left[\begin{array}{l}
0 \\
0
\end{array}\right]^{4}\left(\theta\left[\begin{array}{l}
0 \\
0
\end{array}\right]^{8}+\theta\left[\begin{array}{l}
0 \\
1
\end{array}\right]^{8}+\theta\left[\begin{array}{l}
1 \\
0
\end{array}\right]^{8}\right)\right)\left(\tau_{1}\right) \\
\times\left(\theta\left[\begin{array}{l}
0 \\
0
\end{array}\right]^{4}\left(\theta\left[\begin{array}{l}
0 \\
0
\end{array}\right]^{8}+\theta\left[\begin{array}{l}
0 \\
1
\end{array}\right]^{8}+\theta\left[\begin{array}{l}
1 \\
0
\end{array}\right]^{8}\right)\right)\left(\tau_{1}^{\prime}\right) \\
=\frac{2}{3} f_{21}\left(\tau_{1}\right) \frac{2}{3} f_{21}\left(\tau_{1}^{\prime}\right) .
\end{gathered}
$$

Next we require that the term $f_{21}\left(\tau_{1}\right)$ disappears in the linear combination $\left(\sum a_{i} f_{i}\right)\left(\tau_{1,1}\right)$, that gives

$$
\left(a_{1}\left(\frac{1}{3} f_{21}+\eta^{12}\right)+2 a_{2}\left(\frac{2}{3} f_{21}-\eta^{12}\right)+2 a_{3} \frac{2}{3} f_{21}\right)\left(\tau_{1}^{\prime}\right)=0
$$

for all $\tau_{1}^{\prime} \in \mathbf{H}_{1}$. This gives two linear equations for the $a_{i}$ which have a unique solution, up to scalar multiple:

$$
a_{1}+4 a_{2}+4 a_{3}=0, \quad a_{1}-2 a_{2}=0, \quad \text { hence }\left(a_{1}, a_{2}, a_{3}\right)=\lambda(-4,-2,3) .
$$

A computation shows that $\left(4 f_{1}+2 f_{2}-3 f_{3}\right)\left(\tau_{1,2}\right)=6 \eta^{12}\left(\tau_{1}\right) \eta^{12}\left(\tau_{1}^{\prime}\right)$. Thus we conclude that

$$
\Xi_{8}\left[\begin{array}{c}
00 \\
00
\end{array}\right]:=\theta\left[\begin{array}{c}
00 \\
00
\end{array}\right]^{4}\left(4 \theta\left[\begin{array}{c}
00 \\
00
\end{array}\right]^{12}+2 \sum_{\delta} \theta[\delta]^{12}-3 \theta\left[\begin{array}{c}
00 \\
00
\end{array}\right]^{4} \sum_{\delta} \theta[\delta]^{8}\right) / 6
$$

satisfies the constraints. We will show in [4] that this is the unique modular form on $\Gamma_{2}(1,2)$ satisfying the constraints.

As $\theta\left[\begin{array}{c}00 \\ 00\end{array}\right]^{4} \Xi_{6}\left[\begin{array}{c}00 \\ 00\end{array}\right]$ satisfies the same constraints (with $\Xi_{6}\left[\begin{array}{c}00 \\ 00\end{array}\right]$ the modular form determined by D'Hoker and Phong in [5,6]) we obtain from the uniqueness (or from a direct computation using the methods of [3]) that

$$
\Xi_{6}\left[\begin{array}{c}
00 \\
00
\end{array}\right]=\left(4 \theta\left[\begin{array}{c}
00 \\
00
\end{array}\right]^{12}+2 \sum_{\delta} \theta[\delta]^{12}-3 \theta\left[\begin{array}{c}
00 \\
00
\end{array}\right]^{4} \sum_{\delta} \theta[\delta]^{8}\right) / 6 .
$$

Another formula for this function is:

$$
\Xi_{6}\left[\begin{array}{c}
00 \\
00
\end{array}\right]=-\left(\theta\left[\begin{array}{c}
00 \\
11
\end{array}\right] \theta\left[\begin{array}{c}
01 \\
00
\end{array}\right] \theta\left[\begin{array}{l}
10 \\
01
\end{array}\right]\right)^{4}-\left(\theta\left[\begin{array}{c}
00 \\
01
\end{array}\right] \theta\left[\begin{array}{c}
01 \\
10
\end{array}\right] \theta\left[\begin{array}{c}
11 \\
00
\end{array}\right]\right)^{4}-\left(\theta\left[\begin{array}{c}
00 \\
10
\end{array}\right] \theta\left[\begin{array}{l}
10 \\
00
\end{array}\right] \theta\left[\begin{array}{l}
11 \\
11
\end{array}\right]\right)^{4},
$$

which is the one found by D'Hoker and Phong in [6], to check the equality between the two expressions for $\Xi_{6}\left[\begin{array}{c}00 \\ 00\end{array}\right]$ one can use the classical theta formula. 


\section{The genus three case}

\subsection{Modular forms in genus three}

In case $g=3$, the $8 \Theta[\sigma]$ 's define a holomorphic map

$$
\mathbf{H}_{3} \rightarrow \mathbf{P}^{7}, \quad \tau \longmapsto(\Theta[000](\tau): \ldots: \Theta[111](\tau)) .
$$

The closure of the image of this map is a 6-dimensional projective variety which is defined by a homogeneous polynomial $F_{16}$ (in eight variables) of degree 16 . In particular, the holomorphic function $\tau \mapsto F_{16}(\ldots, \Theta[\sigma](\tau), \ldots)$ is identically zero on $\mathbf{H}_{3}$.

To write down $F_{16}$ we recall the following relation, which holds for all $\tau \in \mathbf{H}_{3}$ :

$$
\begin{aligned}
& r_{1}-r_{2}=r_{3}, \quad \text { with } r_{1}=\prod_{a, b \in \mathbf{F}_{2}} \theta\left[\begin{array}{c}
000 \\
0 a b
\end{array}\right](\tau), \\
& r_{2}=\prod_{a, b \in \mathbf{F}_{2}} \theta\left[\begin{array}{c}
000 \\
1 a b
\end{array}\right](\tau), \quad r_{3}=\prod_{a, b \in \mathbf{F}_{2}} \theta\left[\begin{array}{c}
100 \\
0 a b
\end{array}\right](\tau) .
\end{aligned}
$$

From this we deduce that $2 r_{1} r_{2}=r_{1}^{2}+r_{2}^{2}-r_{3}^{2}$ and thus

$$
r_{1}^{4}+r_{2}^{4}+r_{3}^{4}-2\left(r_{1}^{2} r_{2}^{2}+r_{1}^{2} r_{3}^{2}+r_{2}^{2} r_{3}^{2}\right)
$$

is zero, as function of $\tau$, on $\mathbf{H}_{3}$. Let $F_{16}$ be the homogeneous polynomial, of degree 16 in the $\Theta[\sigma]$ 's, obtained (using the classical theta formula (3.2)) from this polynomial (of degree 8) in the $\theta[\Delta]^{2}$. In [10] it is shown that $F_{16}$ is not zero as a polynomial in the eight $\Theta[\sigma]$. Thus the polynomial $F_{16}$ defines the image of $\mathbf{H}_{3} \rightarrow \mathbf{P}^{7}$.

A computer computation, using once again the classical formula, shows that $F_{16}$ coincides, up to a scalar multiple, with the degree 16 polynomial in the $\Theta[\sigma]$ obtained from

$$
8 \sum_{\Delta} \theta[\Delta]^{16}-\left(\sum_{\Delta} \theta[\Delta]^{8}\right)^{2}
$$

by the classical theta formulas.

\subsection{The functions $F_{i}$}

In analogy with the genus two case, we now want to find a modular form $\Xi_{8}\left[\begin{array}{c}000 \\ 000\end{array}\right]$ of weight 8 on $\Gamma_{3}(1,2)$ which restricts to the 'diagonal' $\Delta_{1,2}$ as

$$
\Xi_{8}\left[\begin{array}{c}
000 \\
000
\end{array}\right]\left(\tau_{1,2}\right)=\Xi_{8}\left[\begin{array}{l}
0 \\
0
\end{array}\right]\left(\tau_{1}\right) \Xi_{8}\left[\begin{array}{c}
00 \\
00
\end{array}\right]\left(\tau_{2}\right)=\left(\theta\left[\begin{array}{l}
0 \\
0
\end{array}\right]^{4} \eta^{12}\right)\left(\tau_{1}\right)\left(\theta\left[\begin{array}{c}
00 \\
00
\end{array}\right]^{4} \Xi_{6}\left[\begin{array}{c}
00 \\
00
\end{array}\right]\right)\left(\tau_{2}\right),
$$

where $\tau_{1,2} \in \mathbf{H}_{3}$ is the block diagonal matrix with entries $\tau_{1} \in \mathbf{H}_{1}$ and $\tau_{2} \in \mathbf{H}_{2}$. An obvious generalization of the functions $f_{i}$ which we considered earlier in Section 3.4 are:

$$
F_{1}:=\theta\left[\begin{array}{c}
000 \\
000
\end{array}\right]^{12}, \quad F_{2}:=\sum_{\Delta} \theta[\Delta]^{12}, \quad F_{3}:=\theta\left[\begin{array}{c}
000 \\
000
\end{array}\right]^{4} \sum_{\Delta} \theta[\Delta]^{8},
$$

where the sum is over the 36 even characteristics $\Delta$ in genus three. The functions $\theta\left[\begin{array}{c}000 \\ 000\end{array}\right]^{4} F_{i}$ are modular forms of weight 8 on $\Gamma_{3}(1,2)$, see Appendix B.3. However, there is no linear combination of these three functions which has the desired restriction. Therefore we introduce another modular form $G\left[\begin{array}{c}000 \\ 000\end{array}\right]$ of weight 8 on $\Gamma_{3}(1,2)$ in the next section. 


\subsection{The modular forms $G[\Delta]$}

For each even characteristic $\Delta$ in $g=3$ we define a modular form $G[\Delta]$ of weight 8 on $\Gamma_{3}(2)$. For a brief introduction to characteristics, quadrics and isotropic subspaces see Appendix A.

An even characteristic $\Delta$ corresponds to a quadratic form

$$
q_{\Delta}: V=\mathbf{F}_{2}^{6} \rightarrow \mathbf{F}_{2}
$$

which satisfies $q_{\Delta}(v+w)=q_{\Delta}(v)+q_{\Delta}(w)+E(v, w)$ where $E(v, w):=\sum_{i=1}^{3}\left(v_{i} w_{3+i}+\right.$ $\left.v_{3+i} w_{i}\right)$. If $\Delta=\left[\begin{array}{c}a b c \\ d e f\end{array}\right]$ then:

$$
q_{\Delta}(v)=v_{1} v_{4}+v_{2} v_{5}+v_{3} v_{6}+a v_{1}+b v_{2}+c v_{3}+d v_{4}+e v_{5}+f v_{6},
$$

where $v=\left(v_{1}, \ldots, v_{6}\right) \in V$, we will also write $v=\left(\begin{array}{l}v_{1} v_{2} v_{3} \\ v_{4} v_{5} v_{6}\end{array}\right)$. Let $Q_{\Delta}=\left\{v \in V: q_{\Delta}(v)=0\right\}$ be the corresponding quadric in $V$.

A Lagrangian (i.e. maximally isotropic subspace) $L \subset V$ is a subspace of $V$ such that $E(v, w)=0$ for all $v, w \in L$ and such that $\operatorname{dim} L=3$. For example, the eight elements $\left(\begin{array}{c}a b c \\ 000\end{array}\right) \in V$ with $a, b, c \in \mathbf{F}_{2}$ form a Lagrangian subspace $L_{0}$ in $V$.

For such a subspace $L$ we define a modular form on a subgroup of $\operatorname{Sp}(6, \mathbf{Z})$ :

$$
P_{L}:=\prod_{Q \supset L} \theta\left[\Delta_{Q}\right]^{2}
$$

here the product is over the even quadrics which contain $L$ (there are eight such quadrics for each $L)$ and $\Delta_{Q}$ is the even characteristic corresponding to $Q$. In case $L=L_{0}$ with

$$
L_{0}:=\left\{\left(v_{1}, \ldots, v_{6}\right) \in V: v_{4}=v_{5}=v_{6}=0\right\}, \quad P_{L_{0}}=\left(r_{1} r_{2}\right)^{2}=\prod_{a, b, c \in \mathbf{F}_{2}} \theta\left[\begin{array}{c}
000 \\
a b c
\end{array}\right]^{2}
$$

with $r_{1}, r_{2}$ as in Section 4.1. The action of $\operatorname{Sp}(6, \mathbf{Z})$ on $V=\mathbf{Z}^{6} / 2 \mathbf{Z}^{6}$ permutes the Lagrangian subspaces $L$, the subgroup $\Gamma_{3}(2)$ acts trivially on $V$. Similarly, the $P_{L}$ are permuted by the action of $\operatorname{Sp}(6, \mathbf{Z})$, see Appendix B.4, and as $\Gamma_{3}(2)$ fixes all $L$ 's, the $P_{L}$ are modular forms on $\Gamma_{3}(2)$ of weight 8 .

For an even characteristic $\Delta$, the quadric $Q_{\Delta}$ contains 30 Lagrangian subspaces. The sum of the $30 P_{L}$ 's, with $L$ a Lagrangian subspace of $Q_{\Delta}$, is a modular form $G[\Delta]$ of weight 8 on $\Gamma_{3}(2)$ :

$$
G[\Delta]:=\sum_{L \subset Q_{\Delta}} P_{L}=\sum_{L \subset Q_{\Delta}} \prod_{Q^{\prime} \supset L} \theta\left[\Delta_{Q^{\prime}}\right]^{2} .
$$

Note that $\theta[\Delta]^{2}$ is one of the factors in each of the 30 products. As the $P_{L}$ are permuted by the action of $\operatorname{Sp}(6, \mathbf{Z})$, also the $G[\Delta]$ are permuted:

$$
G[M \cdot \Delta](M \cdot \tau)=\operatorname{det}(C \tau+D)^{8} G[\Delta](\tau) .
$$

As $\Gamma_{3}(1,2)$ fixes the characteristic $\left[\begin{array}{c}000 \\ 000\end{array}\right]$, the function $G\left[\begin{array}{c}000 \\ 000\end{array}\right]$ is a modular form on $\Gamma_{3}(1,2)$.

\subsection{The restriction}

Now we try to find a linear combination of the functions $\theta\left[\begin{array}{c}000 \\ 000\end{array}\right]^{4} F_{i}, i=1,2,3$ and $G\left[\begin{array}{c}000 \\ 000\end{array}\right]$ which satisfies the third constraint:

$$
\left(\theta\left[\begin{array}{l}
0 \\
0
\end{array}\right]^{4} \eta^{12}\right)\left(\tau_{1}\right)\left(\theta\left[\begin{array}{c}
00 \\
00
\end{array}\right]^{4} \Xi_{6}\left[\begin{array}{c}
00 \\
00
\end{array}\right]\right)\left(\tau_{2}\right)=\left(\theta\left[\begin{array}{c}
000 \\
000
\end{array}\right]^{4}\left(b_{1} F_{1}+b_{2} F_{2}+b_{3} F_{3}\right)+b_{4} G\left[\begin{array}{c}
000 \\
000
\end{array}\right]\right)\left(\tau_{1,2}\right) .
$$


It is easy to see that the theta constants satisfy:

$$
\theta\left[\begin{array}{l}
a b c \\
d e f
\end{array}\right]\left(\tau_{1,2}\right)=\theta\left[\begin{array}{l}
a \\
d
\end{array}\right]\left(\tau_{1}\right) \theta\left[\begin{array}{l}
b c \\
e f
\end{array}\right]\left(\tau_{2}\right)
$$

in particular $\theta\left[\begin{array}{l}a b c \\ d e f\end{array}\right] \mapsto 0$ if $a d=1$. Thus 6 of the 36 even theta constants map to zero, the other $30=3 \times 10$ are uniquely decomposed in the product of two even theta constants for $g=1$ and $g=2$ respectively. Using the results from (3.3), the functions $F_{i}\left(\tau_{1,2}\right)$ are then easy to describe, the function $G\left[\begin{array}{c}000 \\ 000\end{array}\right]\left(\tau_{1,2}\right)$ is determined in Appendix C.3. The restrictions to $\Delta_{1,2} \cong \mathbf{H}_{1} \times \mathbf{H}_{2}$ are:

$$
\begin{gathered}
\left(\theta\left[\begin{array}{c}
000 \\
000
\end{array}\right]^{12}\right)_{\mid \Delta_{1,2}}=\theta\left[\begin{array}{l}
0 \\
0
\end{array}\right]^{12} \theta\left[\begin{array}{l}
00 \\
00
\end{array}\right]^{12} \\
=\left(\frac{1}{3} f_{21}+\eta^{12}\right) \theta\left[\begin{array}{l}
00 \\
00
\end{array}\right]^{12}, \\
\left(\sum_{\Delta} \theta[\Delta]^{12}\right)_{\mid \Delta_{1,2}}=\left(\theta\left[\begin{array}{l}
0 \\
0
\end{array}\right]^{12}+\theta\left[\begin{array}{l}
0 \\
1
\end{array}\right]^{12}+\theta\left[\begin{array}{l}
1 \\
0
\end{array}\right]^{12}\right)\left(\sum_{\delta} \theta[\delta]^{12}\right) \\
=\left(\frac{2}{3} f_{21}-\eta^{12}\right) \sum_{\delta} \theta[\delta]^{12}, \\
\left(\theta\left[\begin{array}{c}
000 \\
000
\end{array}\right]^{4} \sum_{\Delta} \theta[\Delta]^{8}\right)_{\mid \Delta_{1,2}}=\theta\left[\begin{array}{l}
0 \\
0
\end{array}\right]^{4}\left(\theta\left[\begin{array}{l}
0 \\
0
\end{array}\right]^{8}+\theta\left[\begin{array}{l}
0 \\
1
\end{array}\right]^{8}+\theta\left[\begin{array}{l}
1 \\
0
\end{array}\right]^{8}\right) \theta\left[\begin{array}{c}
00 \\
00
\end{array}\right]^{4}\left(\sum_{\delta} \theta[\delta]^{8}\right) \\
=\frac{2}{3} f_{21}\left(\theta\left[\begin{array}{c}
00 \\
00
\end{array}\right]^{4}\left(\sum_{\delta} \theta[\delta]^{8}\right)\right), \\
\times\left(\theta\left[\begin{array}{c}
000 \\
000
\end{array}\right]_{\mid \Delta_{1,2}}=\left(\begin{array}{c}
0 \\
0
\end{array}\right]^{4}\left(\frac{1}{3} f_{21}-\eta^{12}\right)\right) \\
\left.\times\left(\frac{0}{00}\right]^{4}\left(\frac{1}{3} \theta\left[\begin{array}{c}
00 \\
00
\end{array}\right]^{12}+\frac{2}{3} \sum_{\delta} \theta[\delta]^{12}-\frac{1}{2} \theta\left[\begin{array}{c}
00 \\
00
\end{array}\right]^{4} \sum_{\delta} \theta[\delta]^{8}\right)\right) .
\end{gathered}
$$

Thus we found the restriction of $\theta\left[\begin{array}{c}000 \\ 000\end{array}\right]^{4}\left(b_{1} F_{1}+b_{2} F_{2}+b_{3} F_{3}\right)+b_{4} G\left[\begin{array}{c}000 \\ 000\end{array}\right]$ to $\Delta_{1,2}$, note that the function $\theta\left[\begin{array}{l}000 \\ 000\end{array}\right]^{4}$ in front of the $F_{i}$ gives the function $\theta\left[\begin{array}{l}0 \\ 0\end{array}\right]^{4}\left(\tau_{1}\right) \theta\left[\begin{array}{l}00 \\ 00\end{array}\right]^{4}\left(\tau_{2}\right)$. In particular, the restriction has a factor $\theta\left[\begin{array}{l}0 \\ 0\end{array}\right]^{4} \theta\left[\begin{array}{c}00 \\ 00\end{array}\right]^{4}$. In order that this restriction is a multiple of $\theta\left[\begin{array}{l}0 \\ 0\end{array}\right]^{4} \eta^{12}$ we need that the term $f_{21}$ disappears, which leads to the equation

$$
\begin{aligned}
& b_{1} \theta\left[\begin{array}{c}
00 \\
00
\end{array}\right]^{12}+2 b_{2} \sum_{\delta} \theta[\delta]^{12}+2 b_{3} \theta\left[\begin{array}{c}
00 \\
00
\end{array}\right]^{4}\left(\sum_{\delta} \theta[\delta]^{8}\right) \\
& \quad+b_{4}\left(\frac{1}{3} \theta\left[\begin{array}{c}
00 \\
00
\end{array}\right]^{12}+\frac{2}{3} \sum_{\delta} \theta[\delta]^{12}-\frac{1}{2} \theta\left[\begin{array}{c}
00 \\
00
\end{array}\right]^{4} \sum_{\delta} \theta[\delta]^{8}\right)=0 .
\end{aligned}
$$

There is a unique solution (up to scalar multiple):

$$
\left(b_{1}, b_{2}, b_{3}, b_{4}\right)=\mu(4,4,-3,-12) \quad(\mu \in \mathbf{C}) .
$$

With $\mu=1$ and the formula for $\Xi_{6}\left[\begin{array}{c}00 \\ 00\end{array}\right]$ from Section 3.4 one finds: 


$$
\begin{aligned}
& \left(\theta\left[\begin{array}{c}
000 \\
000
\end{array}\right]^{4}\left(4 F_{1}+4 F_{2}-3 F_{3}\right)-12 G\left[\begin{array}{c}
000 \\
000
\end{array}\right]\right)\left(\tau_{1,2}\right) \\
& \quad=\left(\theta\left[\begin{array}{l}
0 \\
0
\end{array}\right]^{4} \eta^{12}\right)\left(\tau_{1}\right)\left(\theta\left[\begin{array}{c}
00 \\
00
\end{array}\right]^{4}\left(8 \theta\left[\begin{array}{c}
00 \\
00
\end{array}\right]^{12}+4 \sum_{\delta} \theta[\delta]^{12}-6 \theta\left[\begin{array}{c}
00 \\
00
\end{array}\right]^{4} \sum_{\delta} \theta[\delta]^{8}\right)\right)\left(\tau_{2}\right) \\
& \quad=12\left(\theta\left[\begin{array}{l}
0 \\
0
\end{array}\right]^{4} \eta^{12}\right)\left(\tau_{1}\right)\left(\theta\left[\begin{array}{c}
00 \\
00
\end{array}\right]^{4} \Xi_{6}\left[\begin{array}{c}
00 \\
00
\end{array}\right]\right)\left(\tau_{2}\right) .
\end{aligned}
$$

Hence the modular form $\Xi_{8}\left[\begin{array}{c}000 \\ 000\end{array}\right]$, of weight 8 on $\Gamma_{3}(1,2)$ defined by

$$
\Xi_{8}\left[\begin{array}{c}
000 \\
000
\end{array}\right]:=\left(\theta\left[\begin{array}{c}
000 \\
000
\end{array}\right]^{4}\left(4 F_{1}+4 F_{2}-3 F_{3}\right)-12 G\left[\begin{array}{c}
000 \\
000
\end{array}\right]\right) / 12
$$

satisfies all the constraints except maybe (iii $)$ (2). To check this last constraint, let $M \cdot\left[\begin{array}{c}000 \\ 000\end{array}\right]=$ $\left[\begin{array}{c}a b c \\ d e f\end{array}\right]$ with $a d=1$, so $a=d=1$. As $G\left[\begin{array}{l}000 \\ 000\end{array}\right](\tau)=\theta^{2}\left[\begin{array}{l}000 \\ 000\end{array}\right](\tau) G^{b}\left[\begin{array}{c}000 \\ 000\end{array}\right](\tau)$ for a holomorphic function $G^{\mathrm{b}}\left[\begin{array}{l}000 \\ 000\end{array}\right], G\left[\begin{array}{l}a b c \\ d e f\end{array}\right](M \cdot \tau)$ is the product of $\theta^{2}\left[\begin{array}{l}a b c \\ d e f\end{array}\right](\tau)$ and a holomorphic function, hence $G\left[\begin{array}{l}a b c \\ d e f\end{array}\right]\left(M \cdot \tau_{1,2}\right)=0$ because $\theta^{2}\left[\begin{array}{l}a b c \\ d e f\end{array}\right]\left(\tau_{1,2}\right)=\theta^{2}\left[\begin{array}{l}1 \\ 1\end{array}\right]\left(\tau_{1}\right) \theta^{2}\left[\begin{array}{l}b c \\ e f\end{array}\right]\left(\tau_{2}\right)=0$.

We conclude that $\Xi_{8}\left[\begin{array}{c}000 \\ 000\end{array}\right]$, defined as above, satisfies all three constraints.

In [4] we will show that it is the only modular form of weight 8 on $\Gamma_{3}(1,2)$ which satisfies the constraints. This then implies that the desired functions $\Xi_{6}[\Delta]$ from [8] indeed do not exist because $G\left[\begin{array}{c}000 \\ 000\end{array}\right]$ is not the product of $\theta\left[\begin{array}{c}000 \\ 000\end{array}\right]^{4}$ with a modular form of weight 6 .

\subsection{The cosmological constant}

In supersymmetric string theories one expects for the cosmological constant to vanish because of perfect cancellation between the positive contribution from bosonic states and the negative one from fermionic states. As a consistency check we will show that our solution for the chiral measure gives a vanishing contribution to the cosmological constant. Like in [6], for type II strings the GSO projections gives $c_{\Delta, \Delta^{\prime}}=1$ and we will prove that

$$
\sum_{\Delta} \mathrm{d} \mu[\Delta]=0, \quad \text { equivalently }\left(\sum_{\Delta} \Xi_{8}[\Delta]\right)(\tau)=0
$$

for all $\tau \in \mathbf{H}_{3}$.

The sum of the 36 functions $\Xi_{8}[\Delta]$ is invariant under $\operatorname{Sp}\left(6, \mathbf{F}_{2}\right)$, hence it is a modular form of weight 8 on $\operatorname{Sp}(6, \mathbf{Z})$. In [4] we will show that it must then be a scalar multiple of $\sum_{\Delta} \theta[\Delta]^{16}$ :

$$
\left(\sum_{\Delta} \Xi_{8}[\Delta]\right)(\tau)=\lambda\left(\sum_{\Delta} \theta[\Delta]^{16}\right)(\tau) \text {. }
$$

The function $\sum_{\Delta} \Xi_{8}[\Delta]$ is given by:

$$
4 \sum_{\Delta} \theta[\Delta]^{16}+4 \sum_{\Delta} \theta[\Delta]^{4}\left(\sum_{\Delta^{\prime}} \epsilon_{\Delta, \Delta^{\prime}} \theta\left[\Delta^{\prime}\right]^{12}\right)-3\left(\sum_{\Delta} \theta[\Delta]^{8}\right)^{2}-12 \sum_{\Delta} G[\Delta],
$$

where the constants $\epsilon_{\Delta, \Delta^{\prime}}= \pm 1$ are determined by the transformation theory of the theta constants.

We will show that $\lambda=0$ by taking first $\tau=\operatorname{diag}\left(\tau_{1}, \tau_{2}, \tau_{3}\right)$ and then let $\tau_{1}, \tau_{2}, \tau_{3} \rightarrow i \infty$. On the theta constants this gives

$$
\theta\left[\begin{array}{l}
a b c \\
\text { def }
\end{array}\right] \longmapsto\left\{\begin{array} { l l } 
{ 1 } & { \text { if } a = b = c = 0 , } \\
{ 0 } & { \text { else } , }
\end{array} \text { hence } \left\{\begin{array}{l}
\sum_{\Delta} \theta[\Delta]^{16} \longmapsto 8, \\
\sum_{\Delta} \theta[\Delta]^{8} \longmapsto 8 .
\end{array}\right.\right.
$$


In the summand $\sum_{\Delta} \theta[\Delta]^{4}\left(\sum_{\Delta^{\prime}} \epsilon_{\Delta, \Delta^{\prime}} \theta\left[\Delta^{\prime}\right]^{12}\right)$ we thus need only consider the terms with $\Delta=$ $\left[\begin{array}{l}0 \\ b\end{array}\right], \Delta^{\prime}=\left[\begin{array}{c}0 \\ b^{\prime}\end{array}\right]$. The terms with $\Delta=\left[\begin{array}{l}0 \\ b\end{array}\right]$ are summands of $\Xi_{8}\left[\begin{array}{l}0 \\ b\end{array}\right](\tau)$. Let $M$ be the symplectic matrix

$$
M=\left(\begin{array}{cc}
I & B \\
0 & I
\end{array}\right), \quad B=\operatorname{diag}\left(b_{1}, b_{2}, b_{3}\right), \quad \text { so } \quad M \cdot\left[\begin{array}{c}
0 \\
b^{\prime}
\end{array}\right]=\left[\begin{array}{c}
0 \\
b+b^{\prime}
\end{array}\right] .
$$

In particular, $M \cdot\left[\begin{array}{l}0 \\ 0\end{array}\right]=\left[\begin{array}{l}0 \\ b\end{array}\right]$ and thus $\Xi_{8}\left[\begin{array}{l}0 \\ b\end{array}\right](\tau)=\Xi_{8}\left[\begin{array}{l}0 \\ 0\end{array}\right]\left(M^{-1} \tau\right)\left(\right.$ note that $\left.\gamma\left(M, M^{-1} \cdot \tau\right)=1\right)$. From the definition of the theta constants as series in 3.1 it is obvious that

$$
\theta\left[\begin{array}{c}
0 \\
b^{\prime}
\end{array}\right]^{4}\left(M^{-1} \cdot \tau\right)=\theta\left[\begin{array}{c}
0 \\
b+b^{\prime}
\end{array}\right]^{4}(\tau)
$$

hence $\epsilon_{\Delta, \Delta^{\prime}}=+1$ if $\Delta=\left[\begin{array}{l}0 \\ b\end{array}\right], \Delta^{\prime}=\left[\begin{array}{c}0 \\ b^{\prime}\end{array}\right]$. Thus we get:

$$
\sum_{\Delta} \theta[\Delta]^{4}\left(\sum_{\Delta^{\prime}} \epsilon_{\Delta, \Delta^{\prime}} \theta\left[\Delta^{\prime}\right]^{12}\right) \longmapsto \sum_{b} \theta\left[\begin{array}{l}
0 \\
b
\end{array}\right]^{4}\left(\sum_{b^{\prime}} \epsilon_{\left[\begin{array}{l}
0 \\
b
\end{array}\right],\left[\begin{array}{c}
0 \\
b^{\prime}
\end{array}\right]} \theta\left[\begin{array}{c}
0 \\
b^{\prime}
\end{array}\right]^{12}\right) \longmapsto 8 \cdot 8=64 .
$$

Finally, each $G[\Delta]$ is a sum of $P_{L}$ 's and each $P_{L}$ is a product of eight distinct theta constants. Thus all $P_{L}$ 's map to zero except for $P_{L_{0}}:=\prod_{d, e, f} \theta\left[\begin{array}{c}000 \\ d e f\end{array}\right]$ which maps to 1 . Note that $L_{0}=$ $\left\{\left(\begin{array}{c}a b c \\ 000\end{array}\right)\right\}$ and that $L_{0} \subset Q_{\Delta}$ iff $\Delta=\left[\begin{array}{c}000 \\ d e f\end{array}\right]$. Thus exactly 8 of the $G[\Delta]$ map to one, and the others map to zero. The constant $\lambda$ can now be determined:

$$
4 \cdot 8+4 \cdot 8^{2}-3 \cdot 64-12 \cdot 8=\lambda \cdot 8 \Longrightarrow \lambda=0,
$$

hence the cosmological constant is zero.

\section{A conjecture of D'Hoker and Phong}

In [7, Section 4.1], D'Hoker and Phong conjecture that:

$$
2^{g} \Psi_{8}(\tau)-\Psi_{4}^{2}(\tau)=0 \quad\left(\forall \tau \in J_{g}\left(\subset \mathbf{H}_{g}\right)\right),
$$

where $J_{g}$ is the closure in $\mathbf{H}_{g}$ of the set of all period matrices $\tau$ of Riemann surfaces of genus $g$, in all genera $g$, where $([7,(3.10)])$

$$
\Psi_{4 k}(\tau):=\sum_{\Delta} \Theta[\Delta]^{8 k}(\tau)
$$

the sum is over the $2^{g-1}\left(2^{g}+1\right)$ even characteristics $\Delta$. They verify this conjecture for $g=1,2$.

The conjecture is actually known to be true for $g \leqslant 4$, due to results of Igusa in [13] which we briefly sketch now. Schottky discovered a modular form $J$ of weight 8 on $\mathbf{H}_{4}$, whose zero locus is known to be $J_{4}$. According to [13, Theorem 1 and its proof], the modular form $J$ is, up to a scalar multiple, equal to

$$
\left(2^{-4} \Psi_{4}(\tau)\right)^{2}-2^{-4} \Psi_{8}(\tau)=-2^{-8}\left(2^{4} \Psi_{8}(\tau)-\Psi_{4}^{2}(\tau)\right)
$$

(use that Igusa's $s_{0,16}=\Psi_{8}$, cf. his definition on p. 356). This verifies the conjecture for $g=4$.

For the case $g=3$ we already verified the conjecture in Section 4.1. Alternatively, one can use the Siegel operator, which is:

$$
\phi(f)\left(\tau_{g-1}\right):=\lim _{\tau_{g} \rightarrow i \infty} f\left(\tau_{g-1,1}\right),
$$


where $\tau_{g-1,1}$ is the matrix in $\mathbf{H}_{g}$ with diagonal blocks $\tau_{g-1} \in \mathbf{H}_{g-1}$ and $\tau_{g} \in \mathbf{H}_{1}$ (and the other coefficients are zero). It is easy to verify that $\phi$ maps $\theta\left[\begin{array}{l}a b c d \\ \text { efgh }\end{array}\right]$ to zero if $d=1$ and else the result is $\theta\left[\begin{array}{l}a b c \\ e f g\end{array}\right]$. Thus applying the Siegel operator to $2^{4} \Psi_{8}-\Psi_{4}^{2}$ we get:

$$
\phi\left(2^{4} \Psi_{8}-\Psi_{4}^{2}\right)\left(\tau_{3}\right)=\left(2^{5} \Psi_{8}-\left(2 \Psi_{4}\right)^{2}\right)\left(\tau_{3}\right)=4\left(2^{3} \Psi_{8}-\Psi_{4}^{2}\right)\left(\tau_{3}\right) .
$$

As any element in $\mathbf{H}_{3}$, viewed as boundary component of $\mathbf{H}_{4}$, is in the closure of $J_{4}$, the modular form $2^{3} \Psi_{8}-\Psi_{4}^{2}$, of weight 8 on $\operatorname{Sp}(6, \mathbf{Z})$ is identically zero on $\mathbf{H}_{3}$.

\section{Conclusions}

In this paper we considered the problem of finding the chiral measure for supersymmetric strings. We have taken the stance that the vacuum to vacuum amplitude should split as in (1). Such a point of view requires a detailed analysis of the geometry of the moduli space of superRiemann surfaces, however, we did not perform such an analysis here.

Instead, we determined the weakest possible constraints that should be obeyed by the measures $\mathrm{d} \mu\left[\Delta^{(3)}\right]$ in order to provide a modular invariant expression for the vacuum to vacuum amplitude. Our constraints are a slight modification of the ones of D'Hoker and Phong in [8]. Using the transformation properties of certain basic functions under suitable subgroups of the modular group, we found that it suffices to consider only one fixed spin structure. This led us to consider modular forms of weight 8 on $\Gamma_{3}(1,2)$ and we explicitly identified the constraints on these modular forms.

The strategy for searching solutions has been to use group representation theory on the space of modular forms. This permitted us to reproduce the genus $g=1,2$ results and to find a solution for the $g=3$ case. Indeed, it happens that our solution is also unique, but this will be proved in [4], together with a systematic study of the modular group representation on modular forms on $\Gamma_{3}(2)$. We also checked that our result is consistent with the vanishing of the cosmological constant, and proved a conjecture of D'Hoker and Phong.

Although the question of the chiral splitting for superstrings in the genus $g=3$ case is open, we think that our results provide some evidence for a positive answer.

\section{Appendix A. Characteristics and quadrics}

We recall the basics about characteristics, in particular their relation to quadratic forms on $V=\mathbf{F}_{2}^{2 g}$, where $\mathbf{F}_{2}:=\mathbf{Z} / 2 \mathbf{Z}$ is the field with two elements (cf. [12, §5.6], but we use the additive convention). We introduce a symplectic form on $V$ (so $E$ is non-degenerate, bilinear and $E(v, v)=0$ for all $v \in V)$,

$$
\begin{aligned}
& E: V \times V \rightarrow \mathbf{F}_{2}, \\
& E(v, w):=v_{1} w_{g+1}+v_{2} w_{g+2}+\cdots+v_{g} w_{2 g}+v_{g+1} w_{1}+\cdots+v_{2 g} w_{g} .
\end{aligned}
$$

We consider the quadratic forms $q$ on $V$ whose associated bilinear form is $E$, that is the maps

$$
q: V \rightarrow \mathbf{F}_{2}, \quad q(v+w)=q(v)+q(w)+E(v, w) .
$$

Note that $q(a x)=a^{2} q(x)=a q(x)$ for $a \in \mathbf{F}_{2}$, i.e., $a=0,1$. If $q, q^{\prime}$ are such quadratic forms then, as $2=0$ in $\mathbf{F}_{2}, q+q^{\prime}$ (defined as usual: $\left(q+q^{\prime}\right)(v)=q(v)+q^{\prime}(v)$ ) is linear in $v:\left(q+q^{\prime}\right)(v+w)=\left(q+q^{\prime}\right)(v)+\left(q+q^{\prime}\right)(w)$ and hence there is a $w \in V$ such that 
$q^{\prime}(v)=q(v)+E(v, w)$ for all $v \in V$. Conversely, if $E$ is associated to $q$ and $w \in V$, then $q^{\prime}$, defined by $q^{\prime}(v)=q(v)+E(v, w)$ also has $E$ as associated bilinear form. Thus, once we fix $q$, for each of the $2^{2 g}$ elements $w \in V$ we have obtained a quadratic form whose associated bilinear form is $E$ and all quadratic forms associated to $E$ are obtained in this way. One verifies easily that for all $\epsilon_{i}, \epsilon_{i}^{\prime} \in \mathbf{F}_{2}$ the function

$$
q(v)=v_{1} v_{g+1}+v_{2} v_{g+2}+\cdots+v_{g} v_{2 g}+\epsilon_{1} v_{1}+\cdots+\epsilon_{g} v_{g}+\epsilon_{1}^{\prime} v_{g+1}+\cdots+\epsilon_{g}^{\prime} v_{2 g}
$$

satisfies $q(v+w)=q(v)+q(w)+E(v, w)$. In this way we obtain $2^{2 g}$ quadratic forms associated to $E$, and thus each of the $2^{2 g}$ quadratic forms associated to $E$ is defined by certain $\epsilon_{i}, \epsilon_{i}^{\prime}, i=$ $1, \ldots, g$. The characteristic associated to $q$ is defined as

$$
\Delta_{q}:=\left[\begin{array}{c}
\epsilon_{1} \epsilon_{2} \ldots \epsilon_{g} \\
\epsilon_{1}^{\prime} \epsilon_{2}^{\prime} \ldots \epsilon_{g}^{\prime}
\end{array}\right], \quad \text { let } \quad e\left(\Delta_{q}\right):=(-1)^{\sum_{i=1}^{g} \epsilon_{i} \epsilon_{i}^{\prime}} \quad(\in\{1,-1\}) .
$$

We say that $\Delta_{q}$ (or $\left.q\right)$ is even if $e\left(\Delta_{q}\right)=+1$ and odd else. One can verify that

$$
e\left(\Delta_{q}\right) 2^{g}=\sum_{v \in V}(-1)^{q(v)} .
$$

For example in case $g=1$ and $q(v)=v_{1} v_{2}$ then $q(v)=0$ for $v=(0,0),(0,1),(1,0)$ and $q(v)=1$ for $v=(1,1)$ so $2 e\left(\left[\begin{array}{l}0 \\ 0\end{array}\right]\right)=3-1=2$; if $q(v)=v_{1} v_{2}+v_{1}+v_{2}$ then $q(v)=1$ except if $v=(0,0)$ so $2 e\left(\left[\begin{array}{l}1 \\ 1\end{array}\right]\right)=1-3=-2$. It follows that $q(v)$ has $2^{g-1}\left(2^{g}+1\right)$ zeroes in $V$ if $\Delta_{q}$ is even and has $2^{g-1}\left(2^{g}-1\right)$ zeroes if $\Delta_{q}$ is odd. Moreover, there are $2^{g-1}\left(2^{g}+1\right)$ even characteristics and $2^{g-1}\left(2^{g}-1\right)$ odd characteristics.

The group $\operatorname{Sp}(2 g, \mathbf{Z})$ acts on $V=\mathbf{Z}^{2 g} /(2 \mathbf{Z})^{2 g}$, the subgroup $\Gamma_{g}(2)$ (see 2.5) acts trivially, so we get an action of the quotient $\operatorname{Sp}(2 g, \mathbf{Z}) / \Gamma_{g}(2) \cong \operatorname{Sp}\left(2 g, \mathbf{F}_{2}\right)$ (cf. [12, V.6, Lemma 25]) on $V$. Let $q: V \rightarrow \mathbf{F}_{2}$ be a quadratic form associated to $E$, then we define a function $\sigma \cdot q$ on $V$ by

$$
(\sigma \cdot q)(v):=q\left(\sigma^{-1} v\right) \quad(v \in V, \sigma \in \operatorname{Sp}(2 g, \mathbf{Z})) .
$$

As $E\left(\sigma^{-1} v, \sigma^{-1} w\right)=E(v, w)$ for $\sigma \in \operatorname{Sp}(2 g, \mathbf{Z})$ and $v, w \in V$, one verifies that also $\sigma \cdot q$ is a quadratic form associated to $E$. Obviously $\sigma \cdot q$ and $q$ have the same number of zeroes, so the parity of $q$ and $\sigma \cdot q$ are the same: $e(\sigma \cdot q)=e(q)$. The group $\operatorname{Sp}(2 g, \mathbf{Z})$ acts transitively on the even and the odd quadrics (cf. [12, V.6, Proposition 3]). If $\Delta_{q}=\left[\begin{array}{c}\epsilon \\ \epsilon^{\prime}\end{array}\right]$ and we write $v=\left(v^{\prime}, v^{\prime \prime}\right)$ as a row vector with $v^{\prime}, v^{\prime \prime} \in \mathbf{F}_{2}^{g}$, then $q(v)=v^{t} v^{\prime \prime}+\epsilon^{t} v^{\prime}+\epsilon^{\prime t} v^{\prime \prime}$. With the formula for $\sigma^{-1}$ from Section B. 2 one then easily verifies that

$$
\Delta_{\sigma \cdot q} \equiv \sigma \cdot \Delta_{q} \bmod 2
$$

with $\sigma \cdot \Delta_{q}$ as in Section 2.3. Thus the interpretation of characteristics as parameters for quadrics associated to $E$ leads to a transformation formula which is exactly the one of the characteristics of the theta constants $\theta[\Delta]$ when we consider the $\Delta$ modulo two.

A subspace $W \subset V$ is isotropic if $E\left(w, w^{\prime}\right)=0$ for all $w, w^{\prime} \in W$. Given a basis $e_{1}, \ldots, e_{k}$ of $W$ it is not hard to see that one can extend it to a symplectic basis $e_{1}, \ldots, e_{2 g}$ of $V$ (so $E\left(e_{i}, e_{j}\right)=0$ unless $|i-j|=g$ and then $\left.E\left(e_{i}, e_{j}\right)=1\right)$. In particular, the group $\operatorname{Sp}(2 g, \mathbf{Z})$ acts transitively on the isotropic subspaces of $V$ of a given dimension. The number of $k$-dimensional isotropic subspaces of $V \cong \mathbf{F}_{2}^{2 g}$ is given by 


$$
\begin{aligned}
& \frac{\left(2^{2 g}-1\right)\left(2^{2 g-1}-2\right)\left(2^{2 g-4}-4\right) \ldots\left(2^{2 g-(k-1)}-2^{k-1}\right)}{\sharp G L\left(k, \mathbf{F}_{2}\right)} \\
& =\frac{\left(2^{2 g}-1\right)\left(2^{2 g-1}-2\right)\left(2^{2 g-2}-4\right) \ldots\left(2^{2 g-(k-1)}-2^{k-1}\right)}{\left(2^{k}-1\right)\left(2^{k}-2\right) \ldots\left(2^{k}-2^{k-1}\right)} \\
& =\frac{\left(2^{2 g}-1\right)\left(2^{2 g-2}-1\right)\left(2^{2 g-4}-1\right)\left(2^{2 g-6}-1\right) \ldots\left(2^{2(g-k)+2}-1\right)}{\left(2^{k}-1\right)\left(2^{k-1}-1\right) \ldots(2-1)},
\end{aligned}
$$

in the numerator we count the ordered $k$-tuples of independent elements $v_{1}, \ldots, v_{k} \in V$ with $E\left(v_{i}, v_{j}\right)=0$ for all $i, j$ : for $v_{1}$ we can take any element in $V-\{0\}$, for $v_{2}$ we can take any element in $\left\langle v_{1}\right\rangle^{\perp} \cong \mathbf{F}_{2}^{2 g-1}$ except $0, v_{1}$, so $v_{2} \in\left\langle v_{1}\right\rangle^{\perp}-\left\langle v_{1}\right\rangle$, next $v_{3} \in\left\langle v_{1}, v_{2}\right\rangle^{\perp}-\left\langle v_{1}, v_{2}\right\rangle$.

If $W_{1}, \ldots, W_{N}$ are the $k$-dimensional isotropic subspaces in an even quadric $Q \subset V$ defined by $q=0$, then $\sigma\left(W_{1}\right), \ldots, \sigma\left(W_{N}\right)$ are the $k$-dimensional isotropic subspaces in the even quadric $\sigma(Q) \subset V$ defined by $\sigma \cdot q=0$, indeed $(\sigma \cdot q)(\sigma v)=q(v)$. In particular, any even quadric in $V$ contains the same number of isotropic subspaces of a given dimension. An even quadric contains a maximal isotropic subspace $L$, for example $L_{0}=\left\{\left(\begin{array}{c}v_{1} \ldots v_{g} \\ 0 \ldots 0\end{array}\right): v_{i} \in \mathbf{F}_{2}\right\}$ is contained in the even quadric $Q$ corresponding to the characteristic $\left[\begin{array}{c}0 \ldots . \\ 0 \ldots 0\end{array}\right]$. An odd quadric does not contain a maximal isotropic subspace however: if $L \subset Q$ were such a subspace, then $\sigma(L)=L_{0}$ for a suitable $\sigma \in \operatorname{Sp}(2 g, \mathbf{Z})$, if $\sigma(Q)$ corresponds to the characteristic $\left[\begin{array}{c}\epsilon \\ \epsilon^{\prime}\end{array}\right]$ then $L_{0} \subset \sigma(Q)$ implies that $\epsilon_{1}=\cdots=\epsilon_{g}=0$, hence the characteristic must be even. On the other hand, an odd quadric does contain an isotropic subspace of dimension $g-1$, for example $W_{0}=\left\{\left(\begin{array}{ccc}v_{1} \ldots v_{g-1} 0 \\ 0 & \ldots & 0\end{array}\right): v_{i} \in \mathbf{F}_{2}\right\}$ is contained in the odd quadric with characteristic $\left[\begin{array}{c}0 \ldots . .01 \\ 0 . .01\end{array}\right]$.

The number of even quadrics which contain a fixed $k$-dimensional isotropic subspace is easy to count: we may assume that the subspace has basis $e_{1}, \ldots, e_{k}$ and then the characteristic of an even quadric containing it is

$$
\left[\begin{array}{cccccc}
0 & \ldots & 0 & \epsilon_{k+1} & \ldots & \epsilon_{g} \\
\epsilon_{1}^{\prime} & \ldots & \epsilon_{k}^{\prime} & \epsilon_{k+1}^{\prime} & \ldots & \epsilon_{g}^{\prime}
\end{array}\right] \text { with } \sum_{i=k+1}^{g} \epsilon_{k+i} \epsilon_{k+i}^{\prime}=0
$$

so one has $2^{k} \cdot 2^{g-k-1}\left(2^{g-k}+1\right)$ such even quadrics. To find the number of $k$-dimensional isotropic subspaces in an even quadric one can now count the pairs $(W, Q)$ of such a subspace $W$ contained in even quadric $Q$ in two ways: first as the product of the number of $W$ with the number of even $Q$ containing a fixed $W$ and second as the product of the number of even quadrics with the number of $k$-dimensional isotropic subspaces in an even quadric. For example the number of pairs $(W, Q)$ of a maximally isotropic subspace in an even quadric in $\mathbf{F}_{2}^{6}$ is $135 \cdot 2^{3}$, and thus the number of such subspaces in a fixed $Q$ is $135 \cdot 2^{3} / 36=30$.

\begin{tabular}{|c|c|c|c|c|c|c|c|c|c|}
\hline \multirow[t]{2}{*}{$g$} & \multicolumn{4}{|c|}{ dimension } & \multirow[t]{2}{*}{$g$} & \multicolumn{4}{|c|}{ dimension } \\
\hline & 1 & 2 & 3 & 4 & & 1 & 2 & 3 & 4 \\
\hline 1 & 3 & & & & 1 & 2 & & & \\
\hline 2 & 15 & 15 & & & 2 & 9 & 6 & & \\
\hline 3 & 63 & 315 & 135 & & 3 & 35 & 105 & 30 & \\
\hline 4 & 255 & 5355 & 11475 & 2295 & 4 & 135 & 1575 & 2025 & 270 \\
\hline
\end{tabular}

For small $g$ we list some of these dimensions in Table 1 on the left, in Table 1 on the right we list the number of $k$-dimensional isotropic subspaces contained in an even quadric.

Table 1 


\section{Appendix B. Transformation theory of theta constants}

\section{B.1. Transformation formula for the $\theta[\Delta]$}

We recall the transformation formula for the functions $\theta[\Delta]$, for an even characteristic $\Delta$ as given in [12, V.1, Corollary].

Let $\Delta=\left[\begin{array}{l}a \\ b\end{array}\right]$ with row vectors $a, b \in \mathbf{Z}^{g}$ and $a_{i}, b_{i} \in\{0,1\}$. We consider the characteristic $m=$ $\left(m^{\prime}, m^{\prime \prime}\right) \in\left(\mathbf{R}^{g}\right)^{2}$ given by $m^{\prime}=a / 2, m^{\prime \prime}=b / 2$. Then $\Theta[\Delta](\tau)=\theta_{m}(\tau)$ and for $\sigma \in \operatorname{Sp}(2 g, \mathbf{Z})$ the transformation formula is:

$$
\theta_{\sigma \cdot m}(\sigma \cdot \tau)=\kappa(\sigma) e^{2 \pi i \phi_{m}(\sigma)} \gamma(\sigma, \tau)^{1 / 2} \theta_{m}(\tau), \quad \sigma=\left(\begin{array}{cc}
A & B \\
C & D
\end{array}\right) \in \operatorname{Sp}(2 g, \mathbf{Z}),
$$

where $\kappa(\sigma)$ is an eight root of unity ([12, V.3, Theorem 3]), $\gamma$ is as in Section 2.1 and

$$
\begin{aligned}
\phi_{m}(\sigma)= & \sum_{k, l=1}^{g} \frac{-1}{8}\left(\left({ }^{t} D B\right)_{k l} a_{k} a_{l}-2\left({ }^{t} B C\right)_{k l} a_{k} b_{l}+\left({ }^{t} C A\right)_{k l} b_{k} b_{l}\right) \\
& +\frac{1}{4}\left(\left({ }^{t} D\right)_{k l} a_{k}-\left({ }^{t} C\right)_{k l} b_{k}\right)\left(A^{t} B\right)_{l l} .
\end{aligned}
$$

\section{B.2. Transformation formula for the $\theta[\Delta]^{4}$}

We apply this formula above to $\theta_{m}^{4}$, so we get the factor $\kappa(\sigma)^{4} \exp \left(8 \pi i \phi_{m}(\sigma)\right) \gamma(\sigma, \tau)$. As $\exp (2 \pi i n)=1$ for integers $n$, we obtain:

$$
e^{8 \pi i \phi_{m}(\sigma)}=(-1)^{a^{t} D B^{t} a+b^{t} C A^{t} b} .
$$

The condition that $\sigma \in \operatorname{Sp}(2 g, \mathbf{Z})$ is that $\sigma E^{t} \sigma=E$ where $E$ has blocks $A=D=0, B=$ $-C=I$ :

$$
\sigma E^{t} \sigma=E \quad \text { iff }\left(\begin{array}{cc}
-B^{t} A+A^{t} B & -B^{t} C+A^{t} D \\
-D^{t} A+C^{t} B & -D^{t} C+C^{t} D
\end{array}\right)=\left(\begin{array}{cc}
0 & I \\
-I & 0
\end{array}\right)
$$

As $E^{-1}=-E$ we find that

$$
\sigma^{-1}=-E^{t} \sigma E, \quad \sigma^{-1}=\left(\begin{array}{cc}
{ }^{t} D & -{ }^{t} B \\
-{ }^{t} C & { }^{t} A
\end{array}\right) .
$$

As $\sigma \in \operatorname{Sp}(2 g, \mathbf{Z})$, also $\sigma^{-1} \in \operatorname{Sp}(2 g, \mathbf{Z})$, thus $\sigma$ satisfies also ${ }^{t} B D-{ }^{t} D B=0$ and ${ }^{t} A C-$ ${ }^{t} C A=0$, that is, ${ }^{t} D B$ and ${ }^{t} C A$ are symmetric matrices. Hence the integers $a_{k} a_{l}, b_{k} b_{l}$ in $a^{t} D B^{t} a+b C A^{t} b$ are multiplied by an even integer if $k \neq l$, and thus they do not contribute to $e^{8 \pi i \phi_{m}(\sigma)}$. In the exponent there remains $\sum_{k}\left(a_{k}^{2}\left({ }^{t} D B\right)_{k k}+b_{k}^{2}\left({ }^{t} C A\right)_{k k}\right)$, but note that $a_{k}^{2} \equiv a_{k} \bmod 2$. For a $g \times g$ matrix $M$, let $\operatorname{diag}(M)$ be the column vector $\left(M_{11}, M_{22}, \ldots, M_{g g}\right)$ of diagonal entries. Then we get the formula:

$$
e^{8 \pi i \phi_{m}(\sigma)}=(-1)^{a \operatorname{diag}\left({ }^{t} D B\right)+b \operatorname{diag}\left({ }^{t} C A\right)} .
$$

Next we consider the case that $\sigma \in \Gamma_{g}(1,2)$. Then also $\sigma^{-1} \in \Gamma_{g}(1,2)$ which implies that the diagonals of ${ }^{t} D B$ and ${ }^{t} C A$ are zero $\bmod 2$, hence we conclude that $e^{8 \pi i \phi_{m}(\sigma)}=1$ for all $\sigma \in \Gamma_{g}(1,2)$.

A final remark is that $\sigma \cdot m$ in [12] is computed in $\mathbf{R}^{2 g}$ whereas we normalize the characteristics modulo vectors in $\mathbf{Z}^{2 g}$ to have coefficients $m_{i}^{\prime}, m_{i}^{\prime \prime} \in\{0,1 / 2\}$. This is justified for the $\theta_{m}^{4}$ by formula $(\theta .2)$ in [12, I.10]. 


\section{B.3. Applications}

Let $\left[\begin{array}{l}0 \\ 0\end{array}\right]=\left[\begin{array}{c}0 \ldots 0 \\ 0 \ldots 0\end{array}\right]$ be a genus $g$ characteristic and let

$$
f_{1}:=\theta\left[\begin{array}{l}
0 \\
0
\end{array}\right]^{12}, \quad f_{2}:=\sum_{\Delta^{(g)}} \theta\left[\Delta^{(g)}\right]^{12}, \quad f_{3}:=\theta\left[\begin{array}{l}
0 \\
0
\end{array}\right]^{4} \sum_{\Delta^{(g)}} \theta\left[\Delta^{(g)}\right]^{8},
$$

where we sum over the even characteristics $\Delta^{(g)}$ in genus $g$. We show that the functions $\theta\left[\begin{array}{l}0 \\ 0\end{array}\right]^{4} f_{i}$, $i=1,2,3$, are modular forms of weight 8 for $\Gamma_{g}(1,2)$.

In the cases $i=1,3$ these functions are polynomials of degree two in the $\theta\left[\Delta^{(g)}\right]^{8}$. The transformation formula for $\theta_{m}^{8}$ (note $\kappa(\sigma)^{8}=1, e^{16 \pi i \phi_{m}(\sigma)}=1$ ), is in our notation:

$$
\theta\left[\Delta^{(g)}\right]^{8}(\sigma \cdot \tau)=\gamma(\sigma, \tau)^{4} \theta\left[\sigma^{-1} \cdot \Delta^{(g)}\right]^{8}(\tau) \quad(\sigma \in \operatorname{Sp}(2 g, \mathbf{Z})) .
$$

Hence the $\theta[\Delta]^{8}$ are permuted by the action of $\sigma \in \operatorname{Sp}(2 g, \mathbf{Z})$, up to the common cocycle $\gamma(\sigma, \tau)^{4}$. In particular, if $\sigma \in \Gamma_{g}(1,2)$ then $\sigma^{-1} \cdot\left[\begin{array}{l}0 \\ 0\end{array}\right]=\left[\begin{array}{l}0 \\ 0\end{array}\right]$ and it follows that the $\theta\left[\begin{array}{l}0 \\ 0\end{array}\right]^{4} f_{i}$, $i=1,3$, are modular forms of weight 8 for $\Gamma_{g}(1,2)$.

In case $i=2$ we use the formula for the $\theta_{m}^{4}$. For $\sigma \in \Gamma_{g}(1,2)$ we have $e^{8 \pi i \phi_{m}(\sigma)}=1$ so in our notation we get:

$$
\theta\left[\Delta^{(g)}\right]^{4}(\sigma \cdot \tau)=\kappa(\sigma)^{4} \gamma(\sigma, \tau)^{2} \theta\left[\sigma^{-1} \cdot \Delta^{(g)}\right]^{4}(\tau) \quad\left(\sigma \in \Gamma_{g}(1,2)\right) .
$$

Hence the $\theta\left[\Delta^{(g)}\right]^{4}$ are permuted by the action of $\sigma \in \Gamma_{g}(1,2)$ up to a common (i.e. independent of $\left.\Delta^{(g)}\right)$ factor $\kappa(\sigma)^{4} \gamma(\sigma, \tau)^{2}$ and these $\sigma$ fix $\left[\begin{array}{l}0 \\ 0\end{array}\right]$. Thus $\theta\left[\begin{array}{l}0 \\ 0\end{array}\right]^{4} f_{2}$ transforms with the factor $\kappa(\sigma)^{16} \gamma(\sigma, \tau)^{8}$, but as $\kappa(\sigma)^{8}=1$ for any $\sigma \in \operatorname{Sp}(2 g, \mathbf{Z})$, this implies that it is a modular form of weight 8 on $\Gamma_{g}(1,2)$.

\section{B.4. Transformation formula for the $P_{L}$}

In Section 4.3 we defined, for a Lagrangian subspace $L$ of $V=\mathbf{F}_{2}^{6}$ the function $P_{L}=$ $\prod_{Q \supset L} \theta\left[\Delta_{Q}\right]^{2}$ where the product is over the eight even quadrics which contain $L$. We will show that these functions are permuted, up to a factor $\gamma(\sigma, \tau)^{8}$, by the action of $\sigma \in \operatorname{Sp}(6, \mathbf{Z})$.

We write representatives in $\mathbf{Z}^{6}$ for the eight elements of $L$ as

$$
\begin{aligned}
L=\left\{p^{(0)}\right. & \left.=\left(\begin{array}{lll}
x_{1}^{(0)} & x_{2}^{(0)} & x_{3}^{(0)} \\
y_{1}^{(0)} & y_{2}^{(0)} & y_{3}^{(0)}
\end{array}\right), \ldots, p^{(7)}=\left(\begin{array}{lll}
x_{1}^{(7)} & x_{2}^{(7)} & x_{3}^{(7)} \\
y_{1}^{(7)} & y_{2}^{(7)} & y_{3}^{(7)}
\end{array}\right)\right\}, \\
& x_{k}^{(j)}, y_{l}^{(j)} \in\{0,1\},
\end{aligned}
$$

and we will assume that $p^{(0)}=\left(\begin{array}{l}0 \\ 0\end{array}\right)$. As $L$ is a subgroup of $\mathbf{F}_{2}^{6}$, it is not hard to see that

$$
\sum_{j=0}^{7} x_{k}^{(j)} \equiv \sum_{j=0}^{7} y_{k}^{(j)} \equiv 0 \bmod 4, \quad \sum_{j=0}^{7} x_{k}^{(j)} x_{l}^{(j)} \equiv \sum_{j=0}^{7} y_{k}^{(j)} y_{l}^{(j)} \equiv \sum_{j=0}^{7} x_{k}^{(j)} y_{l}^{(j)} \equiv 0 \bmod 2
$$

for $k, l=1,2,3$ (use for example $\left(\begin{array}{l}x \\ y\end{array}\right) \mapsto x_{k}$ (or $y_{k}$ ) is a homomorphism of $L \cong \mathbf{F}_{2}^{3}$ to $\mathbf{F}_{2}$ and thus each fiber has either 4 or 8 elements; similarly, the fibers of the homomorphism $L \rightarrow \mathbf{F}_{2}^{2}$, $\left(\begin{array}{l}x \\ y\end{array}\right) \mapsto\left(x_{k}, x_{l}\right)$ contain an even number of elements, etc. $)$. 
Let $Q_{\Delta}$ be one of the quadrics containing $L$ with $\Delta=\left[\begin{array}{l}a \\ b\end{array}\right]$. Then $\Delta^{(j)}:=\left[\begin{array}{l}a+y^{(j)} \\ b+x^{(j)}\end{array}\right]$ is an even characteristic:

$$
\left(a+y^{(j)}\right)^{t}\left(b+x^{(j)}\right)=a^{t} b+x^{(j) t} y^{(j)}+a^{t} x^{(j)}+b^{t} y^{(j)} \equiv 0+q_{\Delta}\left(p^{(j)}\right) \bmod 2,
$$

with $q_{\Delta}$ the quadratic form defining $Q_{\Delta}$; as $L \subset Q_{\Delta}$ we have $q_{\Delta}\left(p^{(j)}\right)=0$ for all $j$ and thus $\left[\begin{array}{c}a+y^{(j)} \\ b+x^{(j)}\end{array}\right]$ is indeed even. Moreover, $L \subset Q_{\Delta^{(j)}}$ because

$$
\begin{aligned}
q_{\Delta^{(j)}}\left(p^{(k)}\right) & =x^{(k) t} y^{(k)}+\left(a+y^{(j)}\right)^{t} x^{(k)}+\left(b+x^{(j)}\right)^{t} y^{(k)} \\
& \equiv q_{\Delta}\left(p^{(k)}\right)+E\left(p^{(j)}, p^{(k)}\right) \equiv 0 \bmod 2 .
\end{aligned}
$$

In this way, given $\Delta$, we get 7 other even characteristics $\Delta^{(j)}$ of quadrics which contain $L$. Therefore the characteristics of the eight even $Q$ with $Q \supset L$ are:

$$
\left[\begin{array}{l}
a^{(j)} \\
b^{(j)}
\end{array}\right], \quad a^{(j)} \equiv a+y^{(j)} \bmod 2, \quad b^{(j)} \equiv b+x^{(j)} \bmod 2, \quad a^{(j)}, b^{(j)} \in\{0,1\} .
$$

It follows that we have the following congruences for the coefficients of the characteristics:

$$
\sum_{j=0}^{7} a_{k}^{(j)} \equiv \sum_{j=0}^{7} b_{k}^{(j)} \equiv 0 \bmod 4, \quad \sum_{j=0}^{7} a_{k}^{(j)} a_{l}^{(j)} \equiv \sum_{j=0}^{7} b_{k}^{(j)} b_{l}^{(j)} \equiv \sum_{j=0}^{7} a_{k}^{(j)} b_{l}^{(j)} \equiv 0 \bmod 2 .
$$

The transformation formula for the theta constants, given in Appendix B.1, shows that

$$
P_{\sigma \cdot L}(\sigma \cdot \tau)=\kappa(\sigma)^{16} e^{4 \pi i \sum_{j=0}^{7} \phi_{m_{j}}(\sigma)} \gamma(\sigma, \tau)^{8} P_{L}(\tau),
$$

where we wrote $m_{j}:=\left(a^{(j)}, b^{(j)}\right) / 2$. As $\kappa(\sigma)$ is an eight root of unity we get $\kappa(\sigma)^{16}=1$. As we observed in Appendix B.2, the matrices ${ }^{t} D B$ and ${ }^{t} C A$ are symmetric. The first term in $2 \sum_{j} \phi_{m_{j}}(\sigma)$ is then

$$
\begin{aligned}
\frac{-1}{4} \sum_{j=0}^{7} \sum_{k, l=1}^{3}\left({ }^{t} D B\right)_{k l} a_{k}^{(j)} a_{l}^{(j)}= & \frac{-1}{4} \sum_{k}^{3}\left({ }^{t} D B\right)_{k k}\left(\sum_{j=0}^{7} a_{k}^{(j)}\right) \\
& -\frac{1}{2} \sum_{k<l}^{3}\left({ }^{t} D B\right)_{k l}\left(\sum_{j=0}^{7} a_{k}^{(j)} a_{l}^{(j)}\right),
\end{aligned}
$$

hence this is an integer. Similarly the third term (with $\left({ }^{t} C A\right)$ ) is an integer. The second term

$$
\frac{-1}{2} \sum_{j=0}^{7} \sum_{k, l=1}^{3}\left({ }^{t} B C\right)_{k l} a_{k}^{(j)} b_{l}^{(j)}=\frac{-1}{2} \sum_{k, l=1}^{3}\left({ }^{t} B C\right)_{k l}\left(\sum_{j=0}^{7} a_{k}^{(j)} b_{l}^{(j)}\right)
$$

is an integer because $\sum_{j} a_{k}^{(j)} b_{l}^{(j)}$ is even for all $k, l$. The last term is also an integer because it is linear in each $a_{k}, b_{l}$ and $\sum_{j} a_{k}^{(j)} \equiv \sum_{j} b_{k}^{(j)} \equiv 0 \bmod 4$.

Again we observe that $\sigma \cdot m$ in [12] is computed in $\mathbf{R}^{2 g}$ whereas we normalize the characteristics modulo vectors in $\mathbf{Z}^{2 g}$ to have coefficients $m_{i}^{\prime}, m_{i}^{\prime \prime} \in\{0,1 / 2\}$. This is justified for the $\theta_{m}^{2}$ by formula $(\theta .2)$ in [12, I.10].

Thus we showed that $e^{4 \pi i \sum_{j=0}^{7} \phi_{m_{j}}(\sigma)}=1$ for all $\sigma$ and it follows that $P_{\sigma(L)}(\sigma \cdot \tau)=$ $\gamma(\sigma, \tau)^{8} P_{L}(\tau)$, as desired. 


\section{Appendix C. The restriction of the $G[\Delta]$ to $\Delta_{1,2}$}

\section{C.1. The restriction of the $P_{L}$ 's to the diagonal}

Now we determine, for each Lagrangian subspace $L \subset V$, the functions $P_{L}\left(\tau_{1,2}\right)$ which are modular forms in both $\tau_{1}$ and $\tau_{2}$.

We already recalled that $\theta\left[\begin{array}{l}a b c \\ d e f\end{array}\right]\left(\tau_{1,2}\right)=\theta\left[\begin{array}{l}a \\ b\end{array}\right]\left(\tau_{1}\right) \theta\left[\begin{array}{l}b c \\ e f\end{array}\right]\left(\tau_{2}\right)$. This 'decomposition' of the characteristic $\Delta=\left[\begin{array}{c}a b c \\ d e f\end{array}\right]$ corresponds to the restriction of the quadratic form $q_{\Delta}$ to the two summands in:

$$
V=l \oplus l^{\perp}, \quad l:=\left\{\left(\begin{array}{c}
000 \\
000
\end{array}\right),\left(\begin{array}{c}
100 \\
000
\end{array}\right),\left(\begin{array}{c}
000 \\
100
\end{array}\right),\left(\begin{array}{c}
100 \\
100
\end{array}\right)\right\}
$$

so $l$ is a two-dimensional symplectic subspace of $V$ (that is, the restriction of the symplectic form $E$ to $l \times l$ is non-degenerate) and its perpendicular is the four dimensional symplectic subspace

$$
l^{\perp}:=\{v \in V: E(v, w)=0 \forall w \in l\}=\left\{\left(\begin{array}{c}
0 a b \\
0 c d
\end{array}\right) \in V: a, \ldots, d \in \mathbf{F}_{2}\right\} .
$$

The restriction of $q_{\Delta}$ to $l$ (respectively $l^{\perp}$ ) is the quadratic form on $\mathbf{F}_{2}^{2}$ (respectively on $\mathbf{F}_{2}^{4}$ ), associated to the characteristic $\left[\begin{array}{l}a \\ d\end{array}\right]$ (respectively $\left[\begin{array}{l}b c \\ e f\end{array}\right]$ ):

$$
\begin{aligned}
& q_{\left[\begin{array}{l}
a \\
d
\end{array}\right]}\left(v_{1}, v_{4}\right)=v_{1} v_{4}+a v_{1}+d v_{4}, \\
& q_{\left[\begin{array}{c}
b c \\
e f
\end{array}\right]}\left(v_{2}, v_{3}, v_{5}, v_{6}\right)=v_{2} v_{5}+v_{3} v_{6}+b v_{2}+c v_{3}+e v_{5}+f v_{6},
\end{aligned}
$$

and $q_{\Delta}=q_{\left[\begin{array}{c}a \\ d\end{array}\right]}+q_{\left[\begin{array}{c}b c \\ e f\end{array}\right]}$.

Let $L$ be a Lagrangian subspace in $V$ and consider the intersection $L \cap l$. As $E$ is nondegenerate on $l$, but is identically zero on $L$, we cannot have $l \subset L$. Thus $\operatorname{dim} L \cap l \leqslant 1$. For dimension reasons, $\operatorname{dim} L \cap l^{\perp} \geqslant 1$ and it is at most two since $L \cap l^{\perp}$ is an isotropic subspace of $l^{\perp}$. We will show that

$$
\operatorname{dim} L \cap l=0 \Longleftrightarrow \operatorname{dim} L \cap l^{\perp}=1, \quad \operatorname{dim} L \cap l=1 \Longleftrightarrow \operatorname{dim} L \cap l^{\perp}=2 .
$$

An example of the first case is

$$
L=\left\langle\left(\begin{array}{c}
000 \\
001
\end{array}\right),\left(\begin{array}{c}
000 \\
110
\end{array}\right),\left(\begin{array}{c}
110 \\
000
\end{array}\right)\right\rangle, \quad L \cap l=\{0\}, \quad L \cap l^{\perp}=\left\langle\left(\begin{array}{c}
000 \\
001
\end{array}\right)\right\rangle,
$$

whereas $L_{0}=\left\{\left(\begin{array}{c}a b c \\ 000\end{array}\right)\right\}$ is an example of the second case.

To prove the assertions, consider the exact sequence

$$
0 \rightarrow L \rightarrow V \stackrel{\phi}{\longrightarrow} L^{*}=\operatorname{Hom}\left(L, \mathbf{F}_{2}\right) \rightarrow 0, \quad \phi(v)\left(v^{\prime}\right):=E\left(v, v^{\prime}\right),
$$

$\left(v \in V, v^{\prime} \in L\right)$, note that $\operatorname{ker}(\phi)=L$ because so $E\left(v, v^{\prime}\right)=0$ for all $v^{\prime} \in L$ implies $v \in L$ by maximality of $L$, hence $\operatorname{dimim}(\phi)=6-3=3=\operatorname{dim} L^{*}$.

In case $L \cap l^{\perp}$ is one-dimensional, that is, $\operatorname{dim}\left(\operatorname{ker}(\phi) \cap l^{\perp}\right)=1$, the subspace $\phi\left(l^{\perp}\right)$ is threedimensional so $\phi\left(l^{\perp}\right)=L^{*}$. Hence for any non-zero $v^{\prime} \in L$ there is a $v \in l^{\perp}$ with $E\left(v, v^{\prime}\right) \neq 0$, and thus $L \cap l=\{0\}$. In case $L \cap l^{\perp}$ is two-dimensional, $\phi\left(l^{\perp}\right)$ is also two-dimensional and hence there is a $w \in L-\{0\}$ such that $E(v, w)=0$ for all $v \in l^{\perp}$. Hence $w \in l$, so in this case $L \cap l=\{0, w\}$. This concludes the proofs of the assertions.

In the first case we claim that there exists an even $q$ such that $L \subset(q=0)$ and such that the restriction of $q$ to $l$ is the odd quadratic form (with characteristic $\left[\begin{array}{l}1 \\ 1\end{array}\right]$ ), hence $P_{L}$ is identically 
zero on the diagonal. To see this, let $w_{0}$ be the unique non-zero element in $l^{\perp} \cap L$. Any $w \in$ $L$ can be written uniquely as $w=w_{l}+w_{p}$ with $w_{l} \in l$ and $w_{p} \in l^{\perp}$. As $L$ is isotropic, we have $0=E\left(w_{0}, w\right)=E\left(w_{0}, w_{p}\right)+E\left(w_{0}, w_{l}\right)$, but $E\left(w_{0}, w_{l}\right)=0$ as $w_{0} \in l^{\perp}$ and $w_{l} \in l$, hence $E\left(w_{0}, w_{p}\right)=0$. Let $q^{\prime}$ be an odd quadratic form on $l^{\perp}$ which is zero in $w_{0}: q^{\prime}\left(w_{0}\right)=0$. Then

$$
q^{\prime}\left(w_{0}+w_{p}\right)=q^{\prime}\left(w_{0}\right)+q^{\prime}\left(w_{p}\right)+E\left(w_{0}, w_{p}\right)=q^{\prime}\left(w_{p}\right) .
$$

In case $w_{p} \neq 0, w_{0}$, we cannot have $q^{\prime}\left(w_{p}\right)=0$, since then $q^{\prime}=0$ would contain the maximal isotropic subspace $\left\langle w_{0}, w_{p}\right\rangle$ of $l^{\perp}$, but odd quadrics do not contain maximal isotropic subspaces. Hence $q^{\prime}\left(w_{p}\right)=q^{\prime}\left(w_{0}+w_{p}\right)=1$ for any $w \in L$. Now let $q^{\prime \prime}$ be the unique odd quadratic form on $l$ and define

$$
q: l \oplus l^{\perp}=V \rightarrow \mathbf{F}_{2}, \quad q\left(l_{0}, l_{p}\right):=q^{\prime \prime}\left(l_{0}\right)+q^{\prime}\left(l_{p}\right) .
$$

Then $q$ is an even quadratic form on $V$ which restricts to the odd quadratic form $q^{\prime \prime}$ on $l$. Moreover, $L \subset(q=0)$ because if $w \in L$ then either $w=0$ and so obviously $q(0)=0$, or $w=w_{0} \in l^{\perp}$ and $q\left(w_{0}\right)=q^{\prime}\left(w_{0}\right)=0$ or $w=w_{l}+w_{p}$ with $w_{l} \neq 0$ and $w_{p} \neq 0$, so $q^{\prime \prime}\left(w_{l}\right)=1$ and $q^{\prime}\left(w_{p}\right)=1$ hence $q(w)=0$. (In the example above one has $L \cap l^{\perp}=\left\langle\left(\begin{array}{c}000 \\ 001\end{array}\right)\right\rangle$ and then $q^{\prime}=q_{\left[\begin{array}{l}1 f \\ 10\end{array}\right]}$, with $f=0,1$ and thus $q=q_{\Delta}$ with $\Delta=\left[\begin{array}{l}11 f \\ 110\end{array}\right]$.) Thus the summands $P_{L}$ of $G[\Delta]$ such that $L \cap l=\{0\}$ are identically zero on the diagonal.

It remains to consider those $L$ such that $L \cap l=\{0, w\}$ for a unique non-zero $w=w_{L} \in l$. In that case $L \cap l^{\perp}$ is a maximal (2-dimensional) isotropic subspace $L_{0}$ of $l^{\perp}$. If $L \subset Q$, the restriction of $Q$ to $l^{\perp}$ contains $L_{0}$. There are four even quadratic forms $q^{\prime}$ on $l^{\perp}$ containing a maximal isotropic subspace, let $\delta_{1}, \ldots, \delta_{4}$ be their characteristics. There are two even quadratic forms $q^{\prime \prime}$ on $l$ with $q^{\prime \prime}(w)=0$, let $\bar{\delta}_{1}, \bar{\delta}_{2}$ be their characteristics. Defining, as before, $q=q^{\prime \prime}+q^{\prime}$ we get $2 \cdot 4=8$ even quadrics which contain $L$. As $L$ is contained in exactly eight quadrics, this implies that these are exactly the quadrics containing $L$ and the product of the squares of the corresponding theta nulls is $P_{L}$. This implies that $P_{L}$ restricts to

$$
P_{L}\left(\tau_{1,2}\right)=\left(\theta\left[\bar{\delta}_{1}\right]^{8} \theta\left[\bar{\delta}_{2}\right]^{8}\right)\left(\tau_{1}\right)\left(\theta\left[\delta_{1}\right]^{4} \theta\left[\delta_{2}\right]^{4} \theta\left[\delta_{3}\right]^{4} \theta\left[\delta_{4}\right]^{4}\right)\left(\tau_{2}\right) .
$$

\section{C.2. The restriction of the $G[\Delta]$ 's to the diagonal}

Now we determine, for each even $\Delta$, the functions $G[\Delta]\left(\tau_{1,2}\right)$ which are modular forms, of weight 8 , in both $\tau_{1}$ and $\tau_{2}$.

Recall that $G[\Delta]$ is a multiple of $\theta[\Delta]^{2}$. In particular, if $\Delta=\left[\begin{array}{c}a b c \\ d e f\end{array}\right]$ and $a d=1$, then $\theta[\Delta]$ restricts to zero on the diagonal $\mathbf{H}_{1} \times \mathbf{H}_{2}$ and thus also $G[\Delta]$ restricts to zero.

As we saw in Appendix C.1, the restriction of $P_{L}$ to $\mathbf{H}_{1} \times \mathbf{H}_{2}$ is non-zero iff $L \cap l=\{0, w\}$ for a non-zero $w \in l$. In that case $L \cap l^{\perp}=\left\{0, w_{p}, w_{p}^{\prime}, w_{p}+w_{p}^{\prime}\right\}$ for some $w_{p}, w_{p}^{\prime} \in l^{\perp}$ is a maximal isotropic subspace of $l^{\perp}$. In particular, $L=(L \cap l) \oplus\left(L \cap l^{\perp}\right)$. Thus these Lagrangian subspaces correspond to pairs of a non-zero point in $Q \cap l$ and a Lagrangian plane in $Q \cap l^{\perp}$.

Let $Q_{\Delta} \subset V$ be the (even) quadric corresponding to $\Delta$ and assume that $a d=0$. Then $Q \cap l$ is an even quadric in $l$ and consists of three points, 0 and $w_{1}, w_{2} \in l$ (for example, if $\left[\begin{array}{l}a \\ d\end{array}\right]=\left[\begin{array}{l}0 \\ 1\end{array}\right]$ then $Q \cap l$ is defined by $v_{1} v_{4}+v_{4}=0$ so consists of the points $\left.\left(\begin{array}{l}v_{1} \\ v_{4}\end{array}\right)=\left(\begin{array}{l}0 \\ 0\end{array}\right),\left(\begin{array}{l}1 \\ 0\end{array}\right),\left(\begin{array}{l}1 \\ 1\end{array}\right)\right)$. The intersection $Q \cap l^{\perp}$ is an even quadric in $l^{\perp}$ and has 10 zeroes, such a quadric contains $2 \cdot 3=6$ Lagrangian subspaces. 
As the Lagrangian subspaces $L \subset V$ such that $P_{L}$ has non-zero restriction to the diagonal correspond to pairs of a non-zero point in $Q \cap l$ and a Lagrangian plane in $Q \cap l^{\perp}$, there are $2 \cdot 6=12$ such subspaces in $Q$.

Using the formula for $P_{L}\left(\tau_{1,2}\right)$ from Appendix C.1, it follows that the restriction of $G[\Delta]$ is given by:

$$
G[\Delta]\left(\tau_{1,2}\right)=\left(\theta\left[\begin{array}{l}
a \\
d
\end{array}\right]^{8} \theta\left[\overline{\delta_{1}}\right]^{8}+\theta\left[\begin{array}{l}
a \\
d
\end{array}\right]^{8} \theta\left[\overline{\delta_{2}}\right]^{8}\right)\left(\tau_{1}\right)\left(\sum_{L^{\prime} \subset Q_{\Delta} \cap l^{\perp}} \prod_{Q^{\prime} \supset L^{\prime}} \theta\left[\delta_{Q^{\prime}}\right]^{4}\right)\left(\tau_{2}\right),
$$

where $\left[\begin{array}{l}a \\ d\end{array}\right],\left[\bar{\delta}_{1}\right],\left[\bar{\delta}_{2}\right]$ are the three even characteristics in $g=1, L^{\prime}$ runs over the six Lagrangian subspaces in $l^{\perp}$ which are contained in $Q_{\Delta}$ and $Q^{\prime}$ runs over the even quadrics in $l^{\perp}$ which contain $L^{\prime}$. In particular, the restriction of a $G[\Delta]$ is a product of a modular form of genus one and one of genus two:

$$
G\left[\begin{array}{l}
a b c \\
d e f
\end{array}\right]\left(\tau_{1,2}\right)=g_{1}\left[\begin{array}{l}
a \\
d
\end{array}\right]\left(\tau_{1}\right) g_{2}\left[\begin{array}{l}
b c \\
e f
\end{array}\right]\left(\tau_{2}\right) .
$$

\section{C.3. The restriction of $G\left[\begin{array}{c}000 \\ 000\end{array}\right]$}

The modular forms of genus one $g_{1}\left[\begin{array}{l}0 \\ 0\end{array}\right]$ and genus two $g_{2}\left[\begin{array}{c}00 \\ 00\end{array}\right]$ are as follows.

$$
\begin{aligned}
g_{1}\left[\begin{array}{l}
0 \\
0
\end{array}\right] & =\theta\left[\begin{array}{l}
0 \\
0
\end{array}\right]^{8} \theta\left[\begin{array}{l}
0 \\
1
\end{array}\right]^{8}+\theta\left[\begin{array}{l}
0 \\
0
\end{array}\right]^{8} \theta\left[\begin{array}{l}
1 \\
0
\end{array}\right]^{8} \\
& =\theta\left[\begin{array}{l}
0 \\
0
\end{array}\right]^{4}\left(\frac{1}{3} f_{21}-\eta^{12}\right),
\end{aligned}
$$

where we used the formulas from Section 3.3. The genus two modular form is:

$$
\begin{aligned}
g\left[\begin{array}{c}
00 \\
00
\end{array}\right]= & \theta\left[\begin{array}{c}
00 \\
00
\end{array}\right]^{4}\left(\left(\theta\left[\begin{array}{c}
00 \\
01
\end{array}\right] \theta\left[\begin{array}{c}
00 \\
10
\end{array}\right] \theta\left[\begin{array}{c}
00 \\
11
\end{array}\right]\right)^{4}+\left(\theta\left[\begin{array}{c}
00 \\
01
\end{array}\right] \theta\left[\begin{array}{c}
10 \\
00
\end{array}\right] \theta\left[\begin{array}{l}
10 \\
01
\end{array}\right]\right)^{4}+\left(\theta\left[\begin{array}{c}
01 \\
00
\end{array}\right] \theta\left[\begin{array}{c}
00 \\
10
\end{array}\right] \theta\left[\begin{array}{l}
01 \\
10
\end{array}\right]\right)^{4}\right. \\
& \left.+\left(\theta\left[\begin{array}{c}
00 \\
11
\end{array}\right] \theta\left[\begin{array}{c}
11 \\
00
\end{array}\right] \theta\left[\begin{array}{c}
11 \\
11
\end{array}\right]\right)^{4}+\left(\theta\left[\begin{array}{c}
01 \\
00
\end{array}\right] \theta\left[\begin{array}{c}
10 \\
00
\end{array}\right] \theta\left[\begin{array}{c}
11 \\
00
\end{array}\right]\right)^{4}+\left(\theta\left[\begin{array}{c}
01 \\
10
\end{array}\right] \theta\left[\begin{array}{c}
10 \\
01
\end{array}\right] \theta\left[\begin{array}{l}
11 \\
11
\end{array}\right]\right)^{4}\right) \\
= & : \theta\left[\begin{array}{c}
00 \\
00
\end{array}\right]^{4} g_{2}^{b}\left[\begin{array}{c}
00 \\
00
\end{array}\right],
\end{aligned}
$$

where $g_{2}^{b}\left[\begin{array}{c}00 \\ 00\end{array}\right]$ is a genus two modular form of weight 6 . A computation, using the methods from [3], that is, using the classical theta formula (3.2) to write the $\theta[\delta]^{2}$ 's in terms of the $\Theta[\sigma]$ 's, shows that

$$
g_{2}^{b}\left[\begin{array}{c}
00 \\
00
\end{array}\right]=\frac{1}{3} \theta\left[\begin{array}{c}
00 \\
00
\end{array}\right]^{12}+\frac{2}{3} \sum_{\delta} \theta[\delta]^{12}-\frac{1}{2} \theta\left[\begin{array}{c}
00 \\
00
\end{array}\right]^{4} \sum_{\delta} \theta[\delta]^{8},
$$

that is, $g_{2}^{b}\left[\begin{array}{c}00 \\ 00\end{array}\right]=\frac{1}{3} f_{1}+\frac{2}{3} f_{2}-\frac{1}{2} f_{3}$ with $f_{i}$ as in Section 3.4.

\section{References}

[1] A.A. Belavin, V.G. Knizhnik, Algebraic geometry and the geometry of quantum strings, Phys. Lett. B 168 (1986) 201-206.

[2] A. Belavin, A. Knizhnik, A. Morozov, A. Perelomov, Two and three loop amplitudes in the bosonic string theory, Phys. Lett. B 177 (1986) 324-328.

[3] S.L. Cacciatori, F. Dalla Piazza, Two loop superstring amplitudes and $S_{6}$ representations, arXiv: 0707.0646, Lett. Math. Phys. (2008), in press.

[4] F. Dalla Piazza, B. van Geemen, Siegel modular forms and finite symplectic groups, in preparation. 
[5] E. D’Hoker, D.H. Phong, Two-loop superstrings I, Main formulas, Phys. Lett. B 529 (2002) 241-255.

[6] E. D'Hoker, D.H. Phong, Two-loop superstrings IV, the cosmological constant and modular forms, Nucl. Phys. B 639 (2002) 129-181.

[7] E. D’Hoker, D.H. Phong, Asyzygies, modular forms, and the superstring measure I, Nucl. Phys. B 710 (2005) 58-82.

[8] E. D’Hoker, D.H. Phong, Asyzygies, modular forms, and the superstring measure II, Nucl. Phys. B 710 (2005) 83-116.

[9] E. D’Hoker, D.H. Phong, Superstrings, super-Riemann surfaces, and supermoduli space, Contribution to the Proceedings of the 'String Theory' Conference, Rome, Italy, June 1988, Rome String Theory 1988, pp. 17-68.

[10] B. van Geemen, G. van der Geer, Kummer varieties and moduli spaces of Abelian varieties, Am. J. Math. 108 (1986) 615-642.

[11] T. Ichikawa, Theta constants and Teichmüller modular forms, J. Number Theory 61 (1996) 409-419.

[12] J. Igusa, Theta Functions, Springer, 1972.

[13] J. Igusa, Schottky's invariant and quadratic forms, in: P.L. Butzer, F. Fehér (Eds.), International Christoffel Symposium proceedings, Birkhäuser, 1981, pp. 352-362.

[14] A. Morozov, Explicit formulae for one, two, three, and four-loop string amplitudes, Phys. Lett. B 184 (1987) 171176.

[15] A. Schwarz, Geometry of Fermionic String, in: Proceedings of the International Congress of Mathematicians, Kyoto, Japan, 1990, pp. 1378-1386.

[16] A. Voronov, A formula for the Mumford measure in superstring theory, Funct. Anal. Appl. 22 (1988) 139-140.

[17] S.L. Cacciatori, F. Dalla Piazza, B. van Geemen, Genus four superstring measures, arXiv: 0804.0457.

[18] S. Grushewsky, Superstring scattering amplitudes in higher genus, arXiv: 0803.3469.

[19] R. Salvati Manni, Remarks on superstring amplitudes in higher genus, arXiv: 0804.0512. 